ARTICLE

\title{
A neural $\mathrm{m}^{6} \mathrm{~A} / \mathrm{Ythdf}$ pathway is required for learning and memory in Drosophila
}

Lijuan Kan ${ }^{1,7}$, Stanislav Ott (2,7, Brian Joseph ${ }^{1,3,7}$, Eun Sil Park ${ }^{1}$, Wei Dai ${ }^{4}$, Ralph E. Kleiner ${ }^{4}$, Adam Claridge-Chang (i) ${ }^{2,5,6}$ \& Eric C. Lai (i) ${ }^{1 \times}$

Epitranscriptomic modifications can impact behavior. Here, we used Drosophila melanogaster to study $\mathrm{N}^{6}$-methyladenosine $\left(\mathrm{m}^{6} \mathrm{~A}\right)$, the most abundant modification of mRNA. Proteomic and functional analyses confirm its nuclear (Ythdc1) and cytoplasmic (Ythdf) YTH domain proteins as major $\mathrm{m}^{6} \mathrm{~A}$ binders. Assays of short term memory in $\mathrm{m}^{6} \mathrm{~A}$ mutants reveal neuralautonomous requirements of $\mathrm{m}^{6} \mathrm{~A}$ writers working via Ythdf, but not Ythdc1. Furthermore, $m^{6} \mathrm{~A} / Y$ thdf operate specifically via the mushroom body, the center for associative learning. We map $\mathrm{m}^{6} \mathrm{~A}$ from wild-type and Mett/3 mutant heads, allowing robust discrimination of Mettl3-dependent $\mathrm{m}^{6} \mathrm{~A}$ sites that are highly enriched in $5^{\prime}$ UTRs. Genomic analyses indicate that Drosophila $\mathrm{m}^{6} \mathrm{~A}$ is preferentially deposited on genes with low translational efficiency and that $\mathrm{m}^{6} \mathrm{~A}$ does not affect RNA stability. Nevertheless, functional tests indicate a role for $\mathrm{m}^{6} \mathrm{~A} / \mathrm{Ythdf}$ in translational activation. Altogether, our molecular genetic analyses and tissuespecific $\mathrm{m}^{6} \mathrm{~A}$ maps reveal selective behavioral and regulatory defects for the Drosophila Mett|3/Ythdf pathway.

\footnotetext{
${ }^{1}$ Department of Developmental Biology, Sloan-Kettering Institute, New York, NY, USA. ${ }^{2}$ Program in Neuroscience and Behavioral Disorders, Duke-NUS Medical School, Singapore, Singapore. ${ }^{3}$ Louis V. Gerstner, Jr. Graduate School of Biomedical Sciences, Memorial Sloan Kettering Cancer Center, New York, NY, USA. ${ }^{4}$ Department of Chemistry, Princeton University, Princeton, NJ, USA. ${ }^{5}$ Institute for Molecular and Cell Biology, A*STAR, Singapore, Singapore. ${ }^{6}$ Department of Physiology, National University of Singapore, Singapore, Singapore. ${ }^{7}$ These authors contributed equally: Lijuan Kan, Stanislav Ott, Brian Joseph.凶email: laie@mskcc.org
} 
bout 45 years ago, pioneering studies led by Seymour Benzer identified dunce, the first learning mutant in any animal $^{1}$, and established Drosophila as an important model to elucidate mechanisms of learning and memory ${ }^{2}$. Although flies execute a broad repertoire of learned behaviors ${ }^{3,4}$, associative odor learning remains the most widely studied type of learning in this organism ${ }^{5}$. In the aversive olfactory-conditioning paradigm, flies are presented with a pair of neutral odors in succession, one in the presence of electric shock and the other without. Subsequently, when flies encounter this odor again later in the absence of shock, the shock-paired odor elicits an avoidance response. A single training trial is sufficient to induce shortterm odor-avoidance memory, which can last several hours ${ }^{6}$. Studies during the past decades identified dozens of proteincoding genes ${ }^{5}$ and a half-dozen microRNAs ${ }^{7}$ that are required for normal short-term memory (STM) formation.

Along with advances in Drosophila memory genetics, substantial progress has been made in deciphering the neuronal anatomy and circuits that underlie memory ${ }^{4,8}$. The mushroom bodies (MB) have been revealed as the higher-order brain center for associative learning 9 ; intrinsic MB neurons called Kenyon cells $(\mathrm{KC})^{10}$ receive olfactory signals from primary olfactory sensory neurons ${ }^{11,12}$ via the antennal lobe ${ }^{13,14}$. As in some vertebrate systems ${ }^{15}$, neuronal ensembles in the Drosophila MB are thought to represent odor-memory engrams that are continuously modified by the animal's experience. More recent studies provided a higher functional resolution of $\mathrm{MB}$ compartments (lobes) with regards to various types of olfactory memory ${ }^{8}$. Moreover, clusters of dopaminergic neurons, such as PPL1 ${ }^{16}$ and PAM $^{17,18}$ were found to innervate distinct MB lobes and provide instructive value to the perceived olfactory stimulus.

In our effort to identify additional factors that regulate memory, we were enticed by the "epitranscriptome", the multitude of modified bases that exist beyond the standard RNA nucleotides. The most abundant and most well-studied internal modification of mRNA is $N^{6}$-methyladenosine $\left(\mathrm{m}^{6} \mathrm{~A}\right)^{19}$. While $\mathrm{m}^{6} \mathrm{~A}$ has been recognized to exist in mRNA since the $1970 \mathrm{~s}^{20,21}$, its functional significance has been elusive until recently. Key advances included (1) techniques to determine individual methylated transcripts, and in particular specific methylated sites, and (2) mechanistic knowledge of factors that install $\mathrm{m}^{6} \mathrm{~A}$ ("writers") and mediate their regulatory consequences ("readers"). The core $\mathrm{m}^{6} \mathrm{~A}$ methytransferase complex acting on mRNA consists of the Mettl3 catalytic subunit and its heterodimeric partner Mettl14. These associate with other proteins that play broader roles in splicing, mRNA processing and gene regulation, but that are collectively required for normal accumulation of $\mathrm{m}^{6} \mathrm{~A}^{19}$.

Downstream of the writers, various readers are sensitive to the presence or absence of $\mathrm{m}^{6} \mathrm{~A}$, and thereby mediate differential regulation by this mRNA modification ${ }^{22}$. The most wellcharacterized readers contain YTH domains, for which atomic insights reveal how a tryptophan-lined pocket selectively binds methylated adenosine and discriminates against unmodified adenosine ${ }^{23-26}$. In addition, some other proteins were proposed as $\mathrm{m}^{6} \mathrm{~A}$ readers, based primarily on preferential in vitro binding to methylated vs. unmethylated RNA probes. In mammals, $\mathrm{m}^{6} \mathrm{~A}$ readers confer diverse regulatory fates onto modified transcripts, including splicing ${ }^{27}$ and nuclear export ${ }^{28}$ via the nuclear reader YTHDC1, and RNA decay via cytoplasmic readers Ythdf1-329-32. Certain YTHDF ${ }^{33-37}$ and YTHDC2 ${ }^{38}$ were also reported to regulate translation via $\mathrm{m}^{6} \mathrm{~A}$ under specific contexts.

Despite intense efforts into $\mathrm{m}^{6} \mathrm{~A}$ mechanisms and genomics using cell systems, genetic analyses of the $\mathrm{m}^{6} \mathrm{~A}$ pathway have only begun in earnest in the past few years, mostly in vertebrates. Notably, many studies have revealed sensitivity of the mammalian nervous system to manipulation of $\mathrm{m}^{6} \mathrm{~A}$ factors ${ }^{39}$. Mutants in writer (Mettl3 and Mettl14), reader (primarily ythdf1), and eraser (FTO) factors have collectively been shown to exhibit aberrant neurogenesis and/or differentiation ${ }^{40-45}$. Moreover, these mutants impact neural function and behavior, including during learning and memory paradigms ${ }^{34,46-50}$. Overall, these observations may reflect some heightened requirements for $\mathrm{m}^{6} \mathrm{~A}$ in neurons, perhaps owing to their unique architectures and/or regulatory needs.

Amongst invertebrates, Caenorhabditis elegans lacks the core $\mathrm{m}^{6} \mathrm{~A}$ machinery ${ }^{51}$, but the presence of a Drosophila ortholog of Mettl3 (originally referred to as IME4) opened this model system ${ }^{52}$. While mammals contain multiple members of both nuclear and cytoplasmic YTH domain families, the fly system is simplified in containing only one of each, referred to as Ythdc1 (YT-521B or CG12076) and Ythdf (CG6422), respectively. Recently, the Soller, Roignant and Lai labs established biochemical, genetic, and genomic foundations for studying the $\mathrm{m}^{6} \mathrm{~A}$ pathway in Drosophila ${ }^{53-55}$. Surprisingly, these studies jointly reported that knockout of all core $\mathrm{m}^{6} \mathrm{~A}$ writer factors in Drosophila is compatible with viability and largely normal exterior patterning. Nevertheless, mutants of Mettl3, Mettl14, and Ythdc1 exhibit a common suite of molecular and phenotypic defects. These include several behavioral abnormalities as well as aberrant splicing of the master female sex determination factor Sex lethal $(S x l)$. The suite of locomotor and postural defects in Drosophila $\mathrm{m}^{6} \mathrm{~A}$ mutants was again consistent with the notion that the nervous system might be especially sensitive.

However, a major open question from these studies concerns the regulatory and biological roles of the sole Drosophila cytoplasmic YTH factor, Ythdf. In contrast to other core $\mathrm{m}^{6} \mathrm{~A}$ factors, we did not previously observe overt defects in our Ythdf mutants, nor did it seem to exhibit robust $\mathrm{m}^{6} \mathrm{~A}$-specific binding activity ${ }^{55}$. Here, we use proteomic analyses to reveal Ythdc1 and Ythdf as the major $\mathrm{m}^{6} \mathrm{~A}$-specific binders in Drosophila, and focused biochemical tests show that Ythdf prefers a distinct sequence context than tested previously. Hypothesizing that the nervous system might exhibit particular needs for the $\mathrm{m}^{6} \mathrm{~A}$ pathway, we utilized a paradigm of aversive olfactory conditioning to reveal an $\mathrm{m}^{6} \mathrm{~A} /$ Ythdf pathway that is important for STM in older animals. We complement these phenotypic data with high-stringency maps of methylated transcript sites from fly heads, and show that $\mathrm{m}^{6} \mathrm{~A}$ does not impact transcript levels but is preferentially deposited on genes with lower translational efficiency. Nevertheless, functional tests reveal that Mettl3/Ythdf can enhance protein output. Finally, we show that physiological Mettl3/Ythdf function is explicitly required within mushroom body neurons to mediate normal conditioned odor memory during aging. Overall, our study provides insights into the in vivo function of this mRNA modification pathway for normal behavior.

\section{Results}

Drosophila Ythdc1 and Ythdf bind $\mathrm{m}^{6} \mathrm{~A}$ in A-rich contexts. In mammals, two general classes of $\mathrm{m}^{6} \mathrm{~A}$-binding proteins ("readers") are recognized, based on whether they contain or lack a YTH domain ${ }^{22}$. Although evidence has been shown for preferential association to $\mathrm{m}^{6} \mathrm{~A}$ vs. A for non-YTH proteins, the YTH domain is the only module for which the structural basis of selective $\mathrm{m}^{6} \mathrm{~A}$ binding is known.

The Drosophila genome encodes single orthologs of nuclear (Ythdc1) and cytoplasmic (Ythdf) YTH factors. We previously tested capacities of their isolated YTH domains to associate preferentially with $\mathrm{m}^{6} \mathrm{~A}$, using RNA probes bearing $\mathrm{GGm}^{6} \mathrm{ACU}$ vs. GGACU contexts ${ }^{55}$. This motif represents the favored binding site for mammalian YTHDC1, which has explicitly been shown to prefer $\mathrm{G}$ and disfavor $\mathrm{A}$ at the -1 position $^{26}$. Of note, however, 

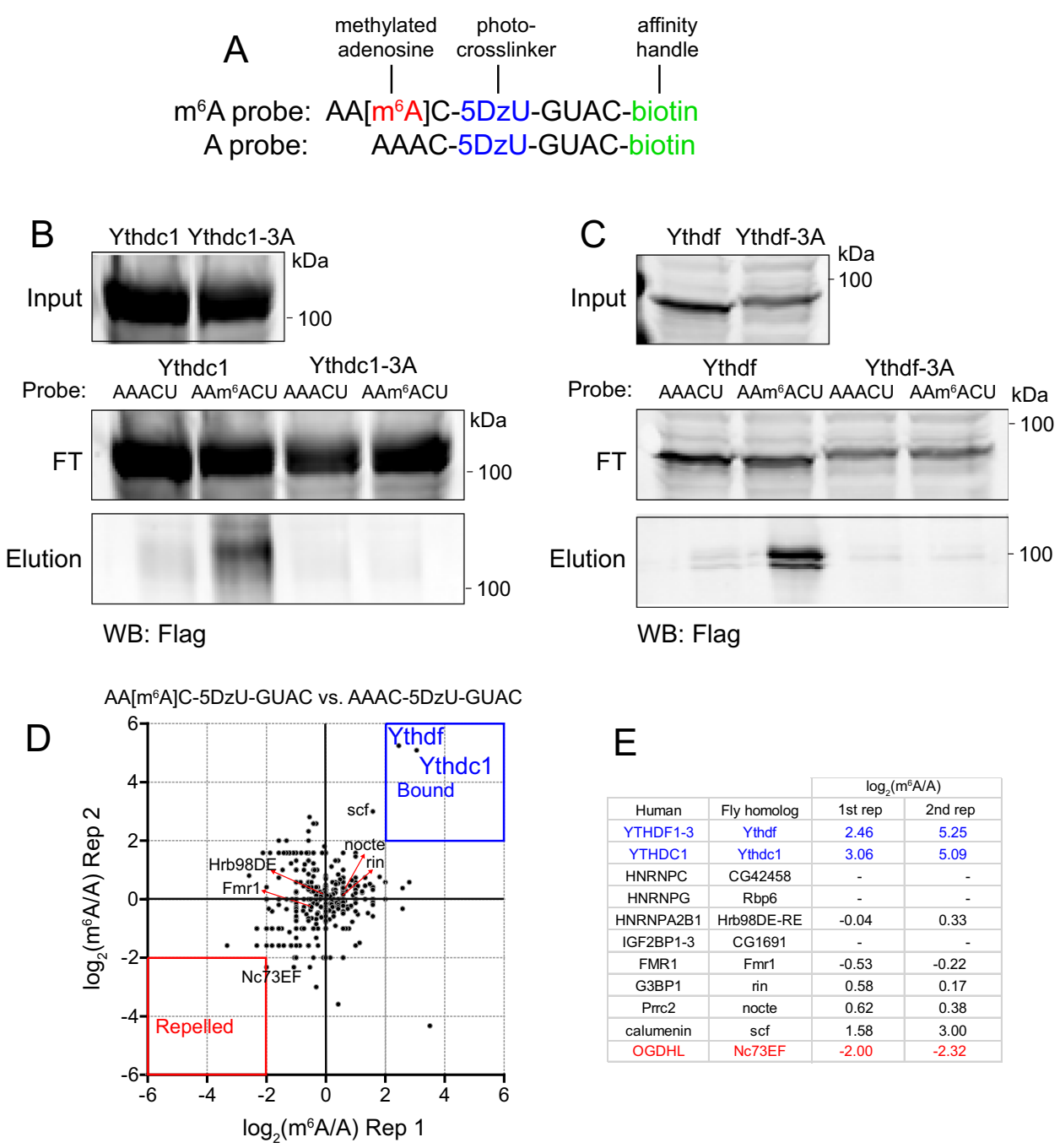

Fig. 1 Ythdc1 and Ythdf are the major $\mathbf{m}^{\mathbf{6}} \mathbf{A}$-binding proteins in Drosophila. A RNA photoaffinity probes used in crosslinking assays. B and $\mathbf{C}$ Both nuclear (Ythdc1) and cytoplasmic (Ythdf) Drosophila YTH factors specifically recognize $\mathrm{m}^{6} \mathrm{~A}$ within the $\mathrm{AAm} \mathrm{m}^{6} \mathrm{ACU}$ context. Point mutations of aromatic residues that line the $\mathrm{m}^{6} \mathrm{~A}$ cage ( $3 \mathrm{~A}$ variants) abolish selective binding to $\mathrm{m}^{6} \mathrm{~A}$ probes. FT Flow through. Shown is one representative result from two repeats. D Proteomic profiling of S2 cells using $\mathrm{m}^{6} \mathrm{~A}$ and A RNA photoaffiinity probes reveals Ythdc1 and Ythdf as the only preferentially bound (reader) proteins, and no strongly repelled proteins were found in these conditions. Background proteins are clustered together around the plot origin; threshold $=2 X$ the interquartile range. Two biological replicates were plotted. E Selected values of fly homologs of mammalian $\mathrm{m}^{6} \mathrm{~A}$ readers and repelled proteins expressed in S2 cells, as well as candidates of novel bound/repelled factors. Source data are provided as a Source Data file and Supplementary Dataset 1.

mammalian YTHDF1 does not share this discriminatory feature $^{25,56}$. We previously observed the YTH domain of Drosophila Ythdc1 exhibits robust and selective binding to this methylated probe, but the corresponding domain of Ythdf had only modest activity. From these tests, it was not clear whether the isolated YTH domain might not be fully functional, or perhaps prefers a distinct target site. We tested both of these notions.

We compared the binding of full-length Ythdc1 and Ythdf proteins to $\mathrm{m}^{6} \mathrm{~A}$ vs. A using biotinylated RNA photoaffinity probes $^{57}$. These probes contain diazirine-modified uridine (5$\mathrm{DzU})$ that can be cross-linked to protein upon UV irradiation (Fig. 1A). We have shown that 5-DzU does not interfere with protein binding at the modified nucleotide, and therefore enables high-efficiency detection of associated proteins ${ }^{57}$. We incubated cell lysates expressing tagged YTH proteins with beads conjugated to GGm ${ }^{6} \mathrm{ACU} / \mathrm{GGACU}$ RNA probes, immunoprecipitated complexes with streptavidin, and performed Western blotting for YTH factors. We observed modestly enhanced association of Ythdc1 to GGm ${ }^{6} \mathrm{ACU}$ vs. GGACU, while Ythdf did not crosslink preferentially to this methylated probe (Supplementary Fig. 1).
As our previous mapping suggested that Drosophila $\mathrm{m}^{6} \mathrm{~A}$ modifications are biased to have upstream adenosines ${ }^{55}$, we next compared AAm ${ }^{6} \mathrm{ACU} / \mathrm{AAACU}$ probes. Interestingly, both Ythdc1 and Ythdf exhibited clearly preferential binding to methylated adenosine in this context (Fig. 1B, C). Next, we tested variants in which three critical tryptophan/leucine residues in the $\mathrm{m}^{6} \mathrm{~A}$-binding pocket were mutated to alanine (Supplementary Fig. 1). Although " $3 \mathrm{~A}$ " mutant proteins accumulated to similar levels as their wild type counterparts, both Ythdc1-3A and Ythdf-3A failed to bind $\mathrm{m}^{6} \mathrm{~A}$ (Fig. $1 \mathrm{~B}, \mathrm{C}$ ), indicating that their specificity for methylated RNA requires intact YTH domains. Thus, Drosophila YTH proteins, in particular Ythdf, may prefer an A-rich context.

Ythdc1 and Ythdf are the dominant Drosophila proteins specifically bound to $\mathrm{AAm}^{\mathbf{6}} \mathrm{ACU}$ probes. Having clarified that both fly YTH factors specifically discriminate between $\mathrm{m}^{6} \mathrm{~A}$ and $\mathrm{A}$, we sought to identify differential binders using an unbiased approach. Proteomic studies in mammalian cells reveal YTH factors as dominant proteins that preferentially associate with 
A

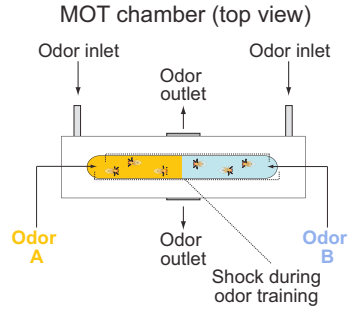

B

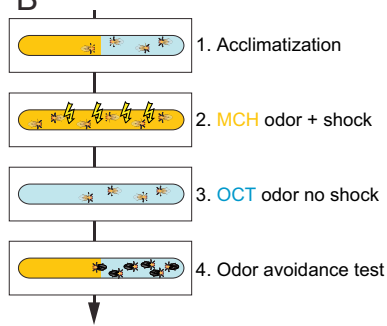

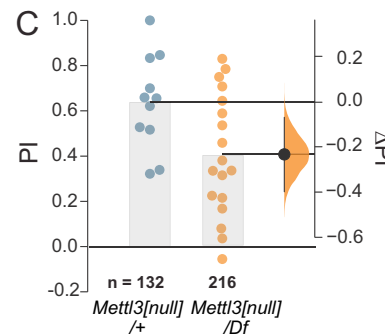

/+

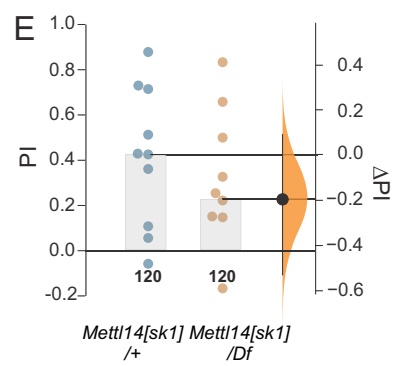

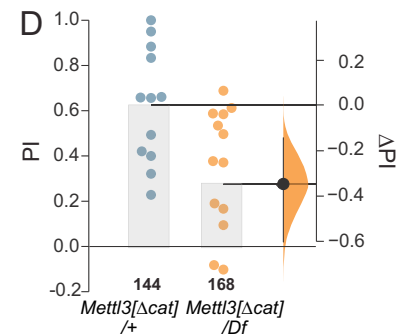

$/ D f$

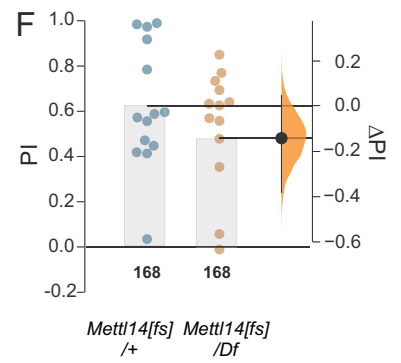

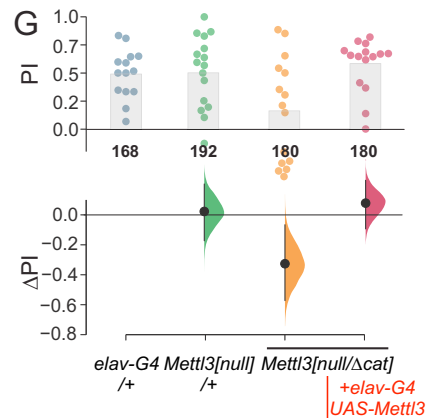

Fig. $2 \mathbf{m}^{\mathbf{6}}$ A pathway is required for short-term learning and memory (STM) in Drosophila. A Schematic of the multifly olfactory training (MOT) chamber apparatus used for behavioral measurements. B Paradigm for shock-associated odor avoidance assay for STM acquisition. C and D Hemizygote null conditions for Mett/3[null] and Mett/3[Acat] both led to STM impairment. Mett/3[null]/Df $(n=216)$ vs. Mett/3[null]/ $+(n=132)=-0.23[95 \mathrm{Cl}-0.4$,

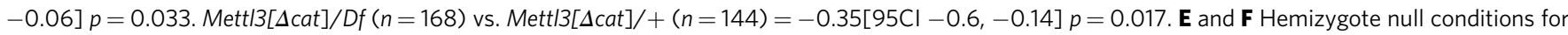
Mett/14[fs] and Mett/14[sk1] led to a mild STM impairment. Mett/14[sk1]/Df $(n=120)$ vs. Mett/14[sk1]/ $+(n=120)=-0.19[95 \mathrm{Cl}-0.52,+0.14] p=0.345$. Mett/14[fs]/Df $(n=168)$ vs. Mett/14[fs]/+ $(n=168)=-0.15[95 \mathrm{Cl}-0.37,+0.06] p=0.505$. G Pan-neuronal expression of UAS-Mett/3 using elav-Gal4 (elav-G4) in MettI3[null] hemizygotes rescued their STM phenotype. Mett/3[null] $/+(n=192)$ vs. elav-G4/w1118 $(n=168)=+0.02[95 \mathrm{Cl}-0.17,+0.21]$

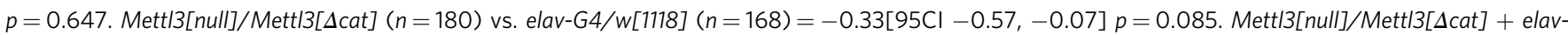
G4 UAS-Mett/3 $(n=180)$ vs. elav-G4/w[1118] $(n=168)=+0.08[95 \mathrm{Cl}-0.1,+0.22] p=0.132$. All assays in C-F were conducted in 20-day-old flies. Bars present mean values. Each dot in the scatter plots represents a PI average of $12 \mathrm{flies}$. All control-test differences are displayed as effect sizes with error curves and $95 \%$ confidence intervals. No null-hypothesis significance testing was performed; two-tailed Mann-Whitney $P$ values are shown for legacy purposes only. For multiple comparisons, several test groups were compared against a common control group. $\mathrm{PI}=$ performance index. Source data are provided as a Source Data file.

$\mathrm{m}^{6} \mathrm{~A}$ compared to unmethylated probes, along with some other proteins (e.g. FMRP and LRPPRC), and reciprocally some factors that are repelled by this modification (e.g. stress granule factors such as G3BP1/2, USP10, CAPRIN1, and RBM42) ${ }^{57,58}$. As well, other methods were used to identify mammalian factors that appear to bind preferentially to $\mathrm{m}^{6} \mathrm{~A}$, such as $\operatorname{Prrc} 2 \mathrm{a}^{59}$ and IGF2BP1-360.

We used our AAm ${ }^{6} \mathrm{ACU} / \mathrm{AAACU}$ RNA photoaffinity probes to pull down endogenous proteins from S2 cell lysates, followed by mass spectrometry. We performed replicate proteomic assays, and plotted the ratios of peptide counts recovered from $\mathrm{m}^{6} \mathrm{~A}$ and A probes (Fig. 1D and Supplementary Dataset 1). These experiments revealed Ythdc1 and Ythdf were strongly and reproducibly enriched with the $\mathrm{m}^{6} \mathrm{~A}$ probe compared to the A probe. By contrast, we did not observe clearly differential association of any other factors, including all fly homologs of other mammalian proteins reported to preferentially bind or be repelled by $\mathrm{m}^{6} \mathrm{~A}^{22}$ (Fig. $\left.1 \mathrm{D}, \mathrm{E}\right)$.

Overall, while conceivable that other target sequences or lysate sources might reveal additional differential binders, we subsequently focused on YTH domain factors as the major direct readers for $\mathrm{m}^{6} \mathrm{~A}$ biology in Drosophila.

Neural autonomous function of $\mathbf{m}^{6} \mathrm{~A}$ supports olfactory learning. The expression of several $\mathrm{m}^{6} \mathrm{~A}$ factors is elevated in the Drosophila nervous system, and mutants of $\mathrm{m}^{6} \mathrm{~A}$ factors are viable, but some exhibit locomotor defects ${ }^{53-55}$. As this suggested preferential sensitivity of the nervous system to $\mathrm{m}^{6} \mathrm{~A}$, we examined phenotypic requirements of neural $\mathrm{m}^{6} \mathrm{~A}$ in greater detail.
Recent studies reported that the $\mathrm{m}^{6} \mathrm{~A}$ pathway is required for learning and memory in mice ${ }^{34,46}$. To investigate whether this is also true for flies, we used a classical aversive conditioning paradigm to test Drosophila $\mathrm{m}^{6} \mathrm{~A}$ mutants for deficits in STM. To obtain time-resolved performance measurements, we employed a conditioning apparatus that we named the multi-fly olfactory trainer (MOT, Fig. 2A and Supplementary Fig. 2). Briefly, during olfactory training one odor is administered in the presence of a shock stimulus while the other odor is subsequently delivered in the absence of foot shock (Fig. 2B). Because shock is innately aversive, Drosophila will associate the odor given in the presence of shock with harm and will tend to avoid it during later encounters. During the test phase the flies are presented with both odors and the avoidance of the conditioned odor is quantified as a measure of aversive shock-odor memory.

We used the respective heterozygotes as controls in following tests. To minimize background genetic effects, a frequent confound of behavioral assays, we compared these to trans-heterozygous or hemizygous (over deficiency) allelic combinations. In young flies, both writer mutants were essentially normal: we observed only a modest STM reduction in 10-day-old Mettl3 hemizygous nulls, while similarly aged Mettll4 mutants showed no impairment (Supplementary Fig. 3). However, at 20 days, both Mettl3[null] and Mettl3 [ $\Delta$ cat] hemizygous nulls displayed a substantially stronger ( $\triangle \mathrm{PI}-0.2$ to -0.3 ) STM impairment (Fig. 2C, D). Consistent with the role of Mettl14 as a cofactor for Mettl3, hemizygote Mettl14[fs], and Mettl14[SK1] mutants also exhibited comparable STM impairments in 20-day-old flies (Fig. 2E, F).

Assays of whole animal mutants did not resolve if the nervous system per se was involved in these behavioral defects. We 
addressed this using tissue-specific knockdown and rescue experiments. We first generated Mettl3[null] hemizygote animals bearing elav-Gal4 and UAS-Mettl3 transgenes, to drive their expression in all neurons. In this genetic background, all nonneuronal cells of the intact animal lack Mettl3. Strikingly, these flies exhibited normal STM (Fig. 2G), providing stringent evidence that the odor avoidance behavioral defect of $\mathrm{m}^{6} \mathrm{~A}$ knockouts is strictly due to a cell-autonomous function of Mettl3 in neurons.

Ythdf, but not Ythdc1, is the functional effector of $\mathrm{m}^{\mathbf{6}} \mathrm{A}$ during STM. We sought to elaborate the regulatory pathway underlying $\mathrm{m}^{6} \mathrm{~A}$ in learning and memory. Prior genetic assays linked $\mathrm{m}^{6} \mathrm{~A}$ writers Mettl3/Mettl14 in a pathway with nuclear reader Ythdc1 for locomotor and gravitaxis behaviors, as well as ovary development ${ }^{53-55}$. By contrast, our Ythdf mutants did not resemble other core $\mathrm{m}^{6} \mathrm{~A}$ mutants, and overall seemed to lack substantial defects in these assays ${ }^{55}$.

The phenotypic discrepancy of these mutants was further emphasized by quantifying their lifespans. While mutations in Mettl3 and Ythdc1 led to severely shortened lifespan ( $>40$ days), loss of Ythdf had only minor effects on lifespan (Fig. 3A-C and Supplementary Fig. $4 \mathrm{a}-\mathrm{c}$ ). As some behavioral effects of the $\mathrm{m}^{6} \mathrm{~A}$ pathway are mediated by the nervous system ${ }^{54}$, we tested the effect of neuronal loss of Mettl3. After validating that a UASMettl3-RNAi transgene was able to deplete both Mettl3 RNA and protein (Supplementary Fig. 5a, b), we tested the consequences of pan-neuronal depletion using elav-Gal4. Compared to controls, loss of neural Mettl3 caused modestly shorter (10 days) lifespan (Supplementary Fig. 4d). These data support the concept of a physiologically important role for nuclear readout of $\mathrm{m}^{6} \mathrm{~A}$ via Ythdc1, with overt, nervous-system effects on longevity.

In light of extensive locomotor defects and short lifespan of Ythdc1 mutants, we were surprised to find that Ythdc1 nulls had normal STM performance at 10 and 20 days of age (Supplementary Fig. 3D and Fig. 3D). We also depleted Ythdc1 using RNAi (Supplementary Fig. 5a), but pan-neuronal knockdown of Ythdc1 using elav-Gal4 in aged flies also did not affect STM (Fig. 3E). Thus, we were prompted to examine mutants of the cytoplasmic reader YTHDF more carefully. Excitingly, Ythdf hemizygotes exhibited age-related STM impairment (Fig. 3F), comparable to Mettl3 mutants. Since Ythdc1 mutants generally phenocopy other defects of $\mathrm{m}^{6} \mathrm{~A}$ writer mutants, these data indicate a division of labor between the Drosophila YTH readers, downstream of $\mathrm{m}^{6} \mathrm{~A}$ writers.

To test whether Ythdf was specifically required in the nervous system, we used a validated RNAi transgene (Supplementary Fig. 5a). 20-day-old elav-Gal4 > UAS-Ythdf[RNAi] flies also exhibited impaired STM (Fig. 3G). Altogether, these data indicate that cytoplasmic readout of $\mathrm{m}^{6} \mathrm{~A}$ by Ythdf is required for normal function of memory-storing neurons in older flies.

Normal locomotor response and olfactory acuity in Mettl3 and Ythdf mutants. Since STM performance depends on locomotion and olfaction, we asked if the defects in Mettl3 and Ythdf mutants were specific to memory-related behavior. We note that a previous study reported adult walking defects in $\mathrm{m}^{6} \mathrm{~A}$ mutants ${ }^{54}$; however, this was measured using a different assay in which the fly wings are removed and animals are provided visual landmarks to promote directional movements (Buridan's paradigm). We examined the locomotor response of $w[1118]$ and $\mathrm{m}^{6} \mathrm{~A}$ mutants before, during, and after a shock stimulus in the MOT. Both Mettl3 and Ythdf locomotor responses to shock were very similar to controls (Supplementary Fig. 6a), indicating that $\mathrm{m}^{6} \mathrm{~A}$ mutants do not exhibit shock sensitivity or locomotion defects in the MOT setup that might affect the memory measurements.

Second, we tested the olfactory acuity of aged $\mathrm{m}^{6} \mathrm{~A}$ mutants. Given a choice between the $\mathrm{MCH}$ odor and clean air (in the absence of conditioning), w[1118], Mettl3, and Ythdf avoidance scores were all comparable (Supplementary Fig. 6b, c). This was true for both the same $\mathrm{MCH}$ concentration used in conditioning experiments, and a four-fold higher concentration. Thus, the olfactory acuity of $\mathrm{m}^{6} \mathrm{~A}$ mutants appears normal.

Neither Mettl3 nor Ythdf can cross-rescue each other's memory defects. We next asked whether overexpression of Ythdf in Mettl3 mutants, or the reciprocal genetic manipulation, would affect STM. Successful rescue could, for example, suggest that the reading function of Ythdf is not fully dependent on Mettl3 methylation, i.e. may somehow involve a parallel pathway. However, Mettl3 nulls supplemented with pan-neuronal Ythdf overexpression did not show STM improvement (Supplementary Fig. 7a). Similarly, overexpression of Mettl3 did not improve the STM impairment in Ythdf mutants (Supplementary Fig. 7b). Beyond serving as stringent negative controls for the cognate rescue experiments (Figs. 2 and 3), these results provide further credence to the notion that a linear, directional Mettl $3 / 14 \rightarrow$ Ythdf pathway underlies $\mathrm{m}^{6} \mathrm{~A}$-mediated function for STM.

Mapping the Mettl3-dependent $\mathbf{m}^{6} \mathrm{~A}$ methylome in Drosophila. To link these brain-function defects to the underlying molecular landscape of RNA methylation, we sequenced $\mathrm{m}^{6} \mathrm{~A}$ sites from polyadenylated transcripts using $\mathrm{miCLIP}^{61}$. Although we previously reported miCLIP datasets from Drosophila embryos ${ }^{55}$, we recognized that there can be background association in such data. Thus, individual sequencing "peaks" need to be interpreted cautiously. To provide a stringent basis to infer the existence of $\mathrm{m}^{6} \mathrm{~A}$ at given sites, we analyzed companion input and miCLIP libraries from dissected heads, which are highly enriched for neurons, comparing wild-type and deletion mutants of Mettl3, which encodes the catalytic methyltransferase subunit essential for mRNA modification (e.g. Fig. 1 and Supplementary Dataset 2).

The miCLIP libraries from Mettl3 mutants proved especially valuable, because they allowed us to distinguish $\mathrm{m}^{6} \mathrm{~A}$-IP loci that were clearly genetically dependent on endogenous Mettl3 (Fig. 4A, Supplementary Fig. 8a). Reciprocally, numerous regions of the transcriptome were significantly enriched in miCLIP libraries compared to input, but whose signals persisted in Mettl3 mutants (Fig. 4B, Supplementary Fig. 8b). These might conceivably represent transcript regions modified by another factor ${ }^{62}$, but cannot at this point be easily distinguished from non-specific pulldown. In general, the Mettl3-independent peaks were globally present in weaker $\mathrm{m}^{6} \mathrm{~A}$ peaks (Fig. 4C), suggesting they are functionally less relevant. Therefore, we applied stringent filtering to focus our attention on the rich set of clearly Mettl3-dependent peaks (Fig. 4A-C). In addition, as we employed strong selection for polyadenylated transcripts for input, we prioritized studies of annotated genes. Altogether, our analyses (see the "Methods" section) yielded 3874 Mettl3-dependent peaks from 1635 genes. Since a subset of these called regions contained clear local minima, we applied PeakSplitter ${ }^{63}$ to arrive at 4686 head $\mathrm{m}^{6} \mathrm{~A}$ peaks (Supplementary Dataset 3).

Drosophila $\mathrm{m}^{6} \mathrm{~A}$ is highly enriched in $5^{\prime}$ UTRs within adenosine-rich contexts. Characterization of Drosophila Mettl3dependent $\mathrm{m}^{6} \mathrm{~A}$ peaks revealed fundamental similarities and differences with $\mathrm{m}^{6} \mathrm{~A}$ patterns in other organisms. Mammalian (e.g. human and mouse) $\mathrm{m}^{6} \mathrm{~A}$ is well-known to dominate at stop codons and $3^{\prime} \mathrm{UTRs}^{19,64}$. In fish, $\mathrm{m}^{6} \mathrm{~A}$ is also highly enriched at 

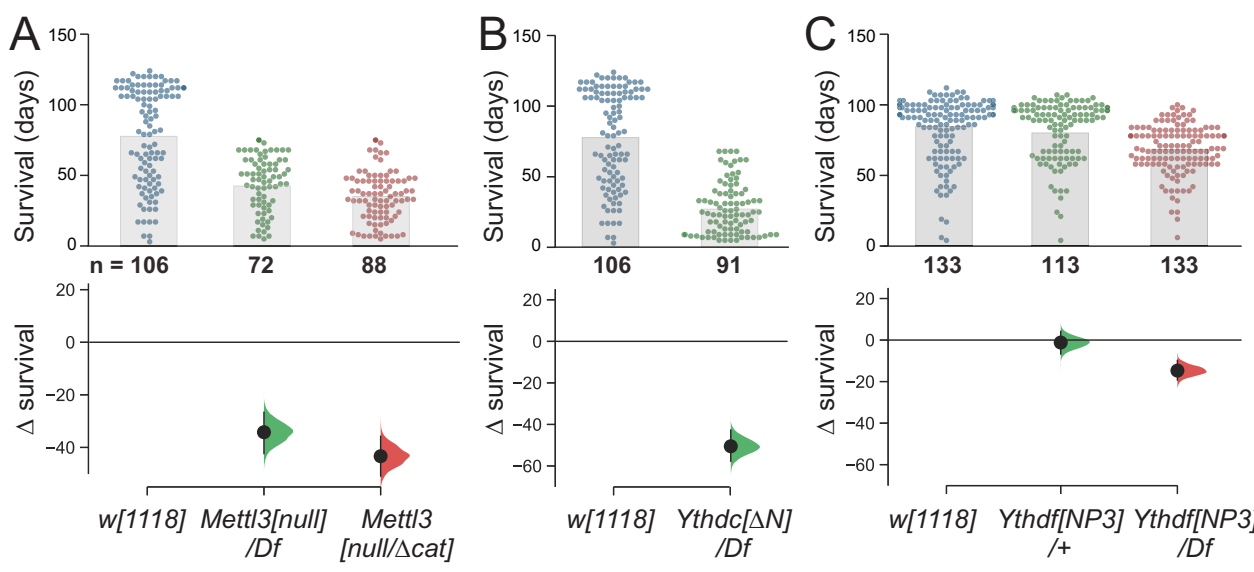

$/ D f$
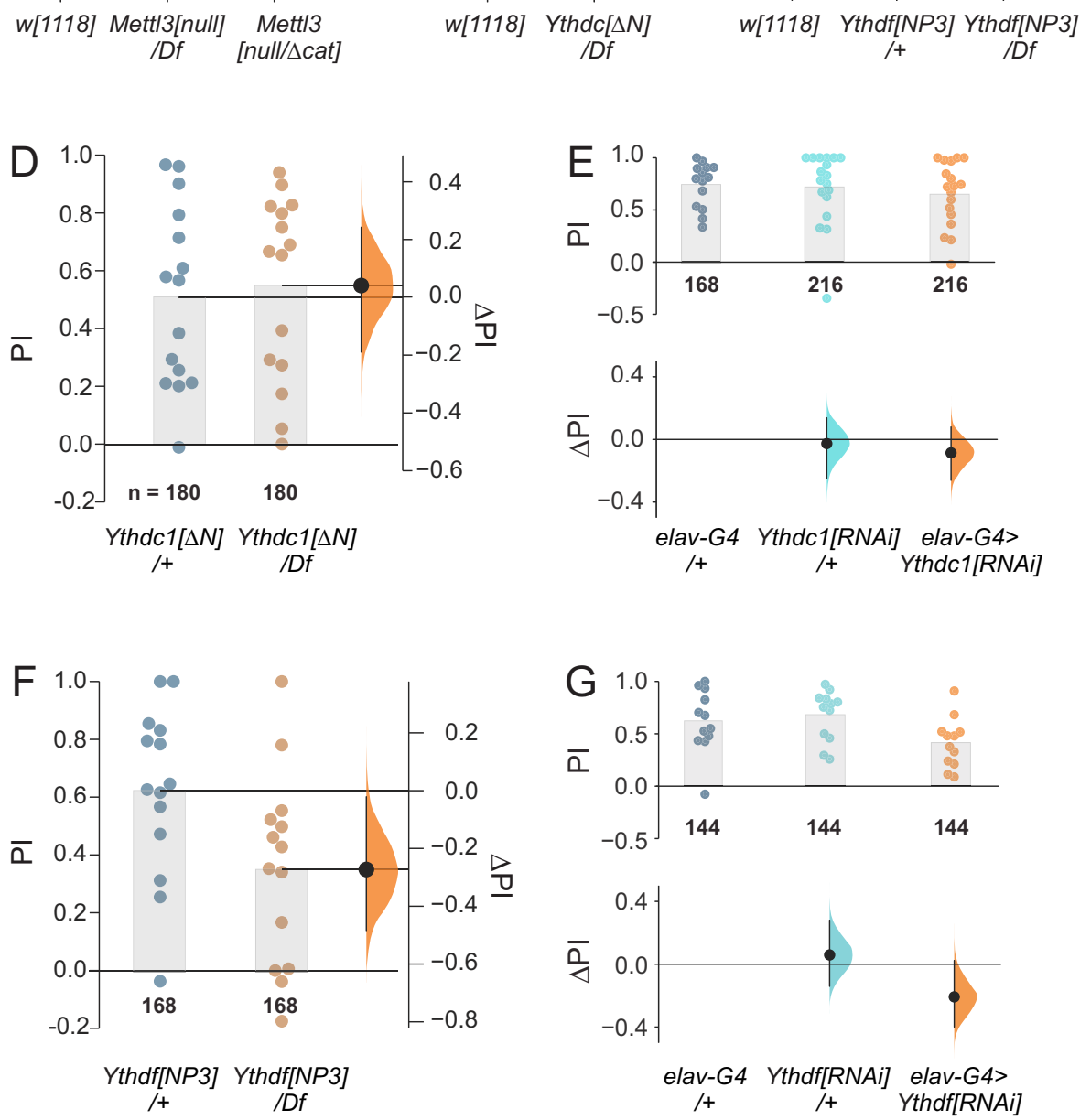

Fig. 3 Ythdf, but not Ythdc1, mediates the role of $\mathbf{m}^{\mathbf{6}} \mathbf{A}$ in Drosophila STM. A-C Lifespan measurements of $\mathrm{m}^{6} \mathrm{~A}$ writer and reader mutants. Mutants of Mettl3 (A) and nuclear reader Ythdc1 (B) exhibit severely shortened lifespan, but mutants of cytoplasmic reader Ythdf (C) shows only a minor lifespan reduction. Mett/3[null]/Df $(n=72)$ vs. $w[1118](n=106)=-34.2[95 \mathrm{Cl}-42.1,-26.31] p<1^{\star} 10^{-4}$. Mett/3[null]/Mettl3[ $\Delta$ cat] $(n=88)$ vs. $w[1118](n=$ $106)=-43.3[95 \mathrm{Cl}-50.66,-35.82] p<1^{\star} 10^{-4}$. Ythdc $[\Delta \mathrm{N}] / \mathrm{Df}(n=91)$ vs. $w[1118](n=106)=-50.5[95 \mathrm{Cl}-57.53,-42.81] p<1^{\star} 10^{-4} . Y$ thdf $[\mathrm{NP3}] /+$ $(n=113)$ vs. $w[1118](n=133)=-1.2[95 \mathrm{Cl}-6.57,+4.24] p=0.431$. Ythdf $[N P 3] / D f(n=133)$ vs. $w[1118](n=133)=-14.7[95 \mathrm{Cl}-19.37,-9.96] p<$ $1^{\star} 10^{-4}$. D-G STM measurements in 20-day flies. D Despite gross behavioral defects and short lifespan, Ythdc1 mutants exhibit normal STM. Ythdc $[\Delta N] / D f$ $(n=180)$ vs. Ythdc1 $[\Delta \mathrm{N}] /+(n=180)=+0.04[95 \mathrm{Cl}-0.18,+0.25] p=0.709$. E Pan-neuronal knockdown of Ythdc1 using elav-Gal4 (elav-G4) also yields normal STM. Ythdc1[RNAi]/+ $(n=216)$ vs. elav-G4/+ $(n=168)=-0.03[95 \mathrm{Cl}-0.25,+0.14] p=0.717$. Elav-G4/Ythdc1[RNAi] ( $n=216)$ vs. elav-G4/+ $(n=168)=-0.09[95 \mathrm{Cl}-0.26,+0.08] p=0.568$. F Ythdf hemizygotes recapitulate age-induced STM impairment seen in $\mathrm{m}^{6} \mathrm{~A}$ writer mutants. Ythdf $[N P 3] / D f(n=168)$ vs. Ythdf $[N P 3] /+(n=168)=-0.27[95 \mathrm{Cl}-0.49,-0.04] p=0.023$. G Pan-neuronal knockdown of Ythdf compromised STM similar to the impairment observed in whole animal Ythdf mutants. Ythdf[RNAi]/ $+(n=144)$ vs. elav-G4/ $+(n=144)=+0.06[95 \mathrm{Cl}-0.14,+0.28] p=0.707$. Elav-G4/Ythdf $[R N A i](n=144)$ vs. elav-G4/ $+(n=144)=-0.21[95 \mathrm{Cl}-0.4,+0.02] p=0.053$. STM assays in D-G were conducted in 20-day-old flies. Bars in D-G represent mean values. All control-test differences are displayed as effect sizes with error curves and $95 \%$ confidence intervals, two-tailed Mann-Whitney $P$ values are shown for legacy purposes only. Source data are provided as a Source Data file. 
A
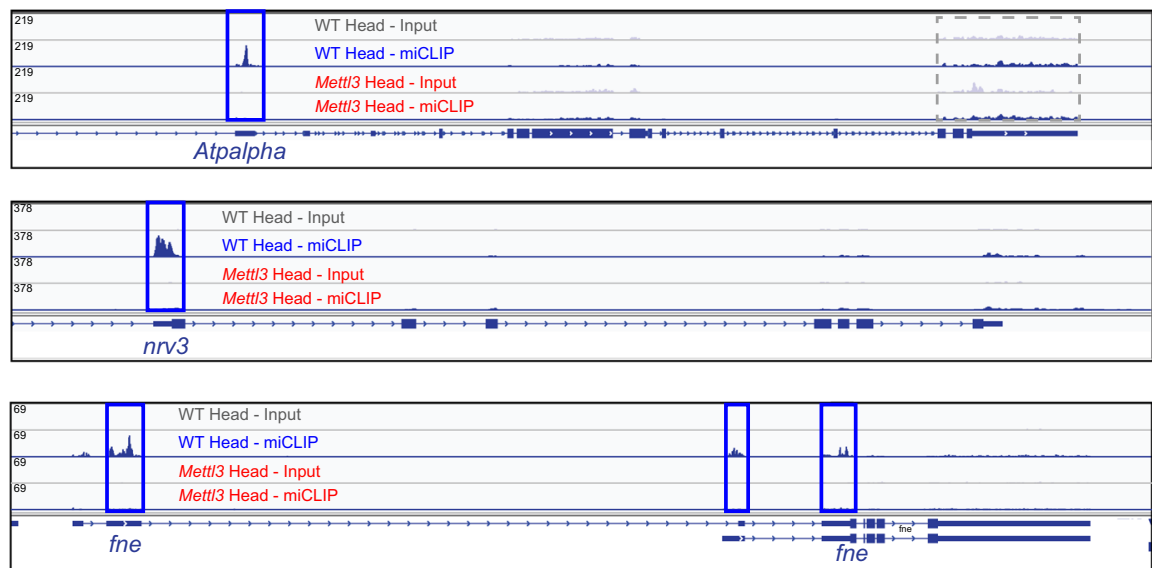

B

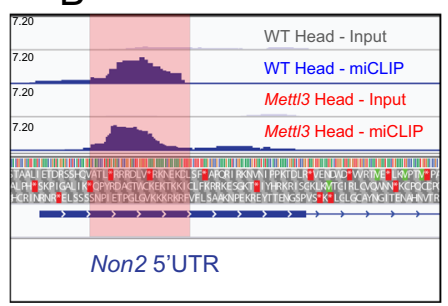

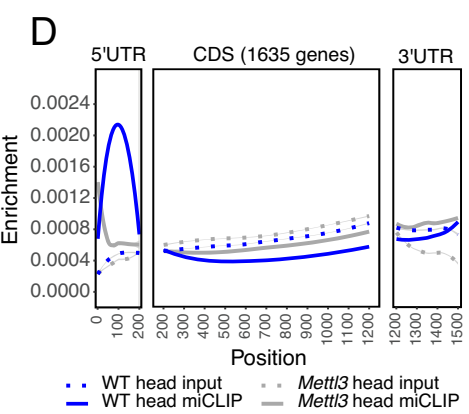

$E_{\text {Metti-dependent Head Peaks }}$

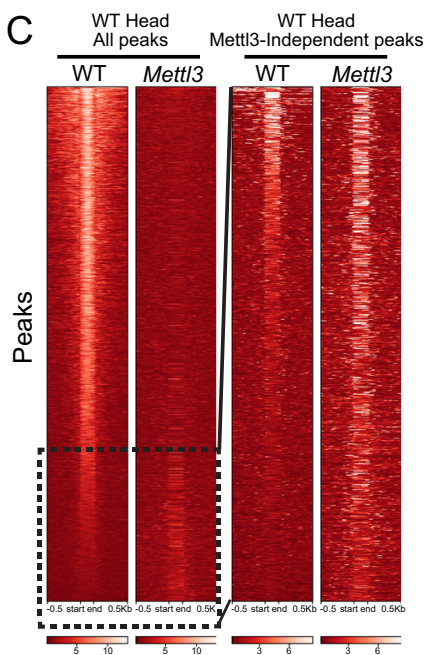

$\mathrm{F}$
F WT Head miCLIP peaks
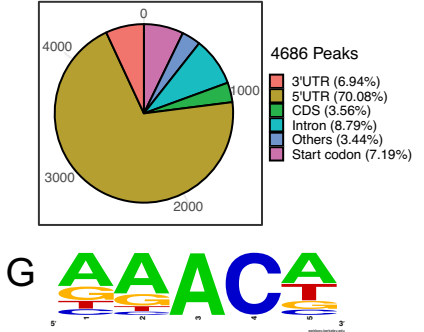

1206 CIMs from top 1000 Mettl3-dependent peaks

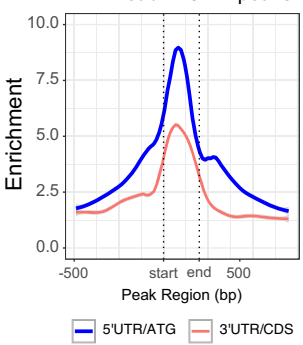

Fig. 4 High stringency mapping of the Drosophila $\mathbf{m}^{\mathbf{6}} \mathbf{A}$ methylome reveals new features. A IGV screenshots of genes that exemplify archetypal $5^{\prime}$ UTR miCLIP enrichment and the utility of wild type vs. Mett/3 mutant comparisons. The IGV tracks above the transcript model depict miCLIP and input libraries from wild type and Mett/3 female heads. Note the 5' UTRs of all three genes (Atpalpha, nrv3, and fne) contain prominent Mettl3-dependent peaks (blue box). Other exonic regions sequenced in miCLIP libraries are not enriched above input libaries (e.g. gray box). B IGV screenshot of Non2 illustrates a $5^{\prime}$ UTR Mett|3-independent peak (red box). C Heatmaps of Mettl3-dependent and -independent $\mathrm{m}^{6} \mathrm{~A}$ peaks. Heatmaps of input normalized miCLIP signals at Mettl3-dependent and independent peaks. Each line represents miCLIP enrichment over input across a MACS2-called peak, as well as 500 nt flanking regions. Heatmaps on the left are all peaks including Mettl3-dependent and independent $\mathrm{m}^{6} \mathrm{~A}$ peaks. Mettl3-independent $\mathrm{m}^{6} \mathrm{~A}$ peaks are $\operatorname{displayed}$ in the right panels. Note that the scales are different for the heatmap panels. Approximate locations of Mettl3-independent $\mathrm{m}^{6} \mathrm{~A}$ peaks within all peaks are indicated by a dashed box. D Metagene profiles of miCLIP and input signals along a normalized transcript using genes that contain high-confidence $\mathrm{m}^{6} \mathrm{~A}$ peaks in head libraries. An overwhelming 5' UTR enrichment is observed. E Metagenes of enrichment at high-confidence m6A peaks that have been group as $5^{\prime}$ UTR/start codon and 3' UTR/CDS regions. Metagenes are produced by averaging signals from input normalized miCLIP from WT head. On average, stronger signals are observed at 5' UTRs/start codons. Peak start and end are specified on the $x$-axis. Dashed lines include the start and end locations of peaks. F Pie chart depicting the fraction of $\mathrm{m}^{6} \mathrm{~A}$ peaks in different transcript segments. G Nucleotide content surrounding CIMs located within the top 1000 Mettl3-dependent $\mathrm{m}^{6} \mathrm{~A}$ peaks in head. Source data are provided as a Source Data file and Supplementary Dataset 3.

stop codons, but the predominant Mettl3-dependent signals localize to $5^{\prime} \mathrm{UTRs}^{65}$. Previous work in Drosophila was conflicting, since low-resolution meRIP-seq suggested mostly CDS modification with a small minority in UTRs ${ }^{54}$, while our prior miCLIP data indicate dominant UTR modifications, preferentially in $5^{\prime}$ UTRs $^{55}$. However, these maps were generated with different technologies, and neither was controlled against mutants.

Our miCLIP data provide a clearer perspective. Strikingly, while found at some level throughout the transcriptome, $\mathrm{m}^{6} \mathrm{~A}$ predominates in $5^{\prime}$ UTRs in Drosophila. This can be observed at numerous individual loci (Fig. 4A and Supplementary Fig. 8) and via miCLIP metagene profiles (Fig. 4D). Overall, while we do observe some Mettl3-dependent coding sequence (CDS) and $3^{\prime}$ UTR miCLIP peaks (Fig. 4E, and Supplementary Fig. 8), these were overall rare, of generally lower ranks than $5^{\prime}$ UTR and start codon peaks (Fig. 4F), and not appreciably enriched in metagene profiles over companion mutant datasets (Fig. 4D).

We examined C-to-T crosslinking-induced mutations following adenosine residues (CIMs), which have been taken to represent individual $\mathrm{m}^{6} \mathrm{~A}$ site in miCLIP data ${ }^{61,66}$. In particular, we focused on CIMs located within Mettl3-dependent $\mathrm{m}^{6} \mathrm{~A}-\mathrm{IP}$ peaks, which we took as bearing high-confidence RNA methylation sites (Supplementary Dataset 4). Within these, the sequence context of CIMs in Drosophila roughly resembles the DRAC context that has been observed in other species ${ }^{19}$. However, while a majority of sites fall into a GGACH context in vertebrates ${ }^{19}, \mathrm{~m}^{6} \mathrm{~A}$ sites in Drosophila prefer AAACD (Fig. 4G), correlating with the preferred binding sites of Ythdc1 and Ythdf in our assays of photocrosslinking-activated $\mathrm{m}^{6} \mathrm{~A}$ probes (Fig. 1).

We validated our map by testing $\mathrm{m}^{6} \mathrm{~A}-\mathrm{IP}$ to IgG-IP samples for enrichment of $\mathrm{m}^{6} \mathrm{~A}$ target transcripts using rt-qPCR (Supplementary Fig. 9). We validated a number of top $\mathrm{m}^{6} \mathrm{~A}$ targets (e.g. aqz, Syx1A, fl(2)d, prosap, pum, futsch, gish) from whole female fly RNA (Fig. 5A). Still, recognizing that $\mathrm{m}^{6} \mathrm{~A}-\mathrm{RIP}-\mathrm{qPCR}$ evaluates the presence of entire transcripts in pulldowns, we performed parallel experiments from Mettl3[null] female flies. All of these binding events, even loci with very modest enrichment in wild type (e.g. sky, Fig. 5A), were found to be Mettl3-dependent. By contrast, control loci lacking $\mathrm{m}^{6} \mathrm{~A}$ peaks (fwe and CG7970) showed very little $\mathrm{m}^{6} \mathrm{~A}$-dependent IP signals, and these were 

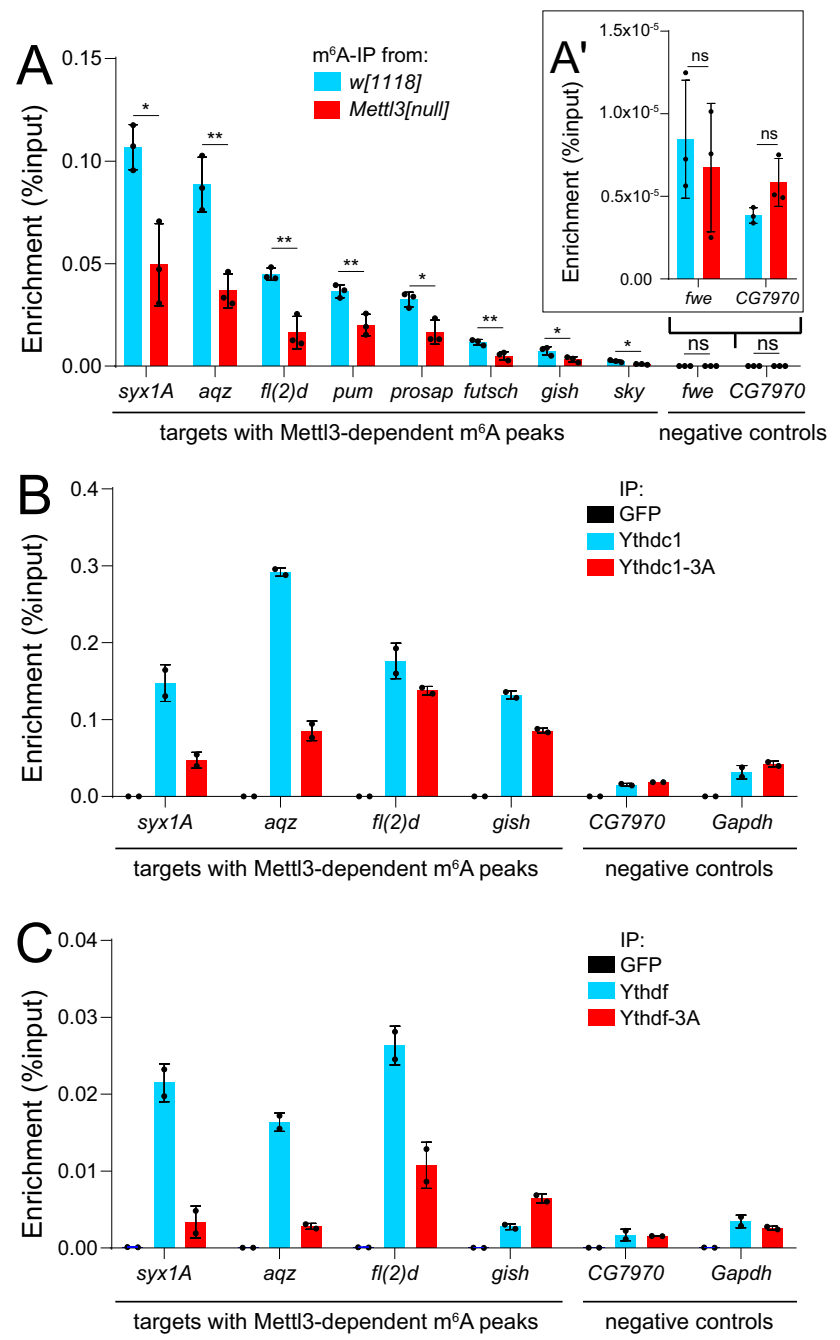

Fig. 5 Validation of Drosophila $\mathbf{m}^{6} \mathbf{A}$ targets and their association with readers. A-A $\mathbf{A}^{\prime} \mathrm{m}^{6} \mathrm{~A}-\mathrm{RIP}-\mathrm{qPCR}$ validation of $\mathrm{m}^{6} \mathrm{~A}$ transcripts in $w[1118]$ control and Mett/3[null] knockout whole female flies. Error bars, mean $\pm \mathrm{SD}$; $n=3$ biological replicates. Two-tailed $t$-test, ${ }^{*} p<0.05,{ }^{\star \star} p<0.01$. syx $1 \mathrm{~A}$ $p=0.012$, aqz $p=0.005$, fl(2)d $p=0.004$, pum $p=0.009$, prosap $p=$ 0.016 , futsch $p=0.009$, gish $p=0.044$, sky $p=0.010$. n.s. non-significant, fwe $p=0.604, C G 7970 p=0.085$. B and C RIP-qPCR of $m^{6} \mathrm{~A}$ targets in S2$S$ cells and transfected Ythdc1 (B) or Ythdf (C) constructs shows specific pulldown of several targets relative to GFP-RIP control, and the association of several of these is compromised by mutation of the YTH domain (3A versions). Shown is one representative result from three independent repeats. Source data are provided as a Source Data file.

unaltered in Mettl3[null] samples (Fig. 5A'). These data provide stringent validation of our $\mathrm{m}^{6} \mathrm{~A}$ maps.

Overall, our high-quality miCLIP data from the Drosophila head reveals that the position of $\mathrm{m}^{6} \mathrm{~A}$ in this species appears distinct amongst metazoans (highly $5^{\prime}$ UTR specific) and occurs within a distinct adenosine-rich context.

Drosophila YTH factors associate with $\mathrm{m}^{6} \mathrm{~A}$ targets in a YTHdependent manner. We next assessed association of $\mathrm{m}^{6} \mathrm{~A}$ targets with YTH factors using transfected constructs in S2-S cells, a derivative of S2 cells lacking viruses ${ }^{67}$. Although overexpression may affect the localization properties of YTH domain proteins ${ }^{31}$, we showed that ectopic Ythdc1 and Ythdf localize to the nucleus and cytoplasm of cultured cells, respectively ${ }^{55}$. In these tests, it is also relevant to consider that we are evaluating the association of the test proteins and target RNAs, which may or may not occur directly through the modified nucleotides. However, we can compare these to YTH-" $3 \mathrm{~A}$ " point-mutant counterparts that disrupt $\mathrm{m}^{6} \mathrm{~A}$ selectivity (Fig. 1).

We immunoprecipitated tagged YTH wild-type or " $3 \mathrm{~A}$ " mutant factors and performed $\mathrm{qPCR}$ for validated $\mathrm{m}^{6} \mathrm{~A}$ targets or negative control transcripts. By comparison to control GFP-IP, we observed preferential binding of Ythdc1/Ythdf on multiple $\mathrm{m}^{6} \mathrm{~A}$ targets, compared to non $-\mathrm{m}^{6} \mathrm{~A}$ transcripts (Fig. $5 \mathrm{~B}, \mathrm{C}$ ). By testing companion " $3 \mathrm{~A}$ " mutant factors, we gained evidence for direct association of $\mathrm{YTH}$ factors on $\mathrm{m}^{6} \mathrm{~A}$ targets. However, a clear picture of target selectivity did not emerge (Fig. 5B, C). Syx1A and aqz exhibited the most clearly differential association between wt and 3A forms of both Ythdcl and Ythdf. We observed potentially selective association with other loci, in that gish was preferentially bound only by wt Ythdc1 while $f(2) d$ was preferentially bound only by wt Ythdf.

We bear in mind these were ectopic experiments, and thus cannot rule out non-physiological associations. Even though we observed many cases of YTH-dependent target association, both YTH-3A proteins still exhibited apparent enrichment compared to GFP. If these mutant YTH proteins are still capable of incorporating into RNA granules, this may conceivably indicate indirect interactions with transcripts. Nevertheless, these data provide evidence that YTH domain proteins, including Ythdf, associate with specific $\mathrm{m}^{6} \mathrm{~A}$ target transcripts via their $\mathrm{m}^{6} \mathrm{~A}$ binding pocket in Drosophila cells.

$\mathrm{m}^{6} \mathrm{~A}$ does not globally influence mRNA levels in Drosophila. There is diverse literature on linking mammalian YTHDF homologs to RNA decay and/or translational activation, while the function of Drosophila $\mathrm{m}^{6} \mathrm{~A} / \mathrm{Ythdf}$ has been little studied. The only prior study integrated MeRIP-seq peaks from S2R+ cells with RNA-seq data from $\mathrm{m}^{6} \mathrm{~A}$ pathway depletions, and concluded that $\mathrm{m}^{6} \mathrm{~A}$ correlated with slightly elevated levels of target mRNAs at steady state $\mathrm{e}^{54}$. With our high-stringency $\mathrm{m}^{6} \mathrm{~A}$ map from heads, we generated RNA-seq data from one- and three-week old heads using Mettl3, Ythdf heterozygotes, and transheterozygotes. The heterozygote samples provide matched genetic backgrounds for comparison, and the temporal series assesses CNS stages including an advanced setting during which behavioral phenotypes were apparent (Figs. 2 and 3).

Transcriptome analyses revealed scores of differentially expressed genes in one- and three-week old mutants (Supplementary Dataset 5), a majority of which were uniquely misexpressed (Supplementary Fig. 10a, b). Most affected genes were not found to be common between $\mathrm{m}^{6} \mathrm{~A}$ writer (Mettl3) and reader (Ythdf) mutants, although there were mild changes that gradually increased with tissue age (Supplementary Fig. 10c and d). Thus, there did not appear to be a clear signature of $\mathrm{m}^{6} \mathrm{~A} /$ Ythdf regulation revealed by bulk gene expression.

We examined this more closely by directly examining the behavior of $\mathrm{m}^{6} \mathrm{~A}$ targets. We reasoned that targets with systematically higher levels of methylation - that is, genes with increasing proportions of methylated transcripts-would be more sensitive to loss of the $\mathrm{m}^{6} \mathrm{~A}$ pathway. However, while our miCLIP libraries provide Mettl3-dependent peaks and single nucleotide resolution mapping of $\mathrm{m}^{6} \mathrm{~A}$ sites in the transcriptome, it is not possible to infer overall methylation levels. A solution to this limitation, grouping targets by number of sites/ peaks, has been adopted by others ${ }^{31,68}$ and proposes that targets with increasing numbers of peaks/sites may have more individual transcripts with at least one $\mathrm{m}^{6} \mathrm{~A}$ modification. Therefore, we binned genes by numbers of Mettl3-dependent $\mathrm{m}^{6} \mathrm{~A}$ peaks. 

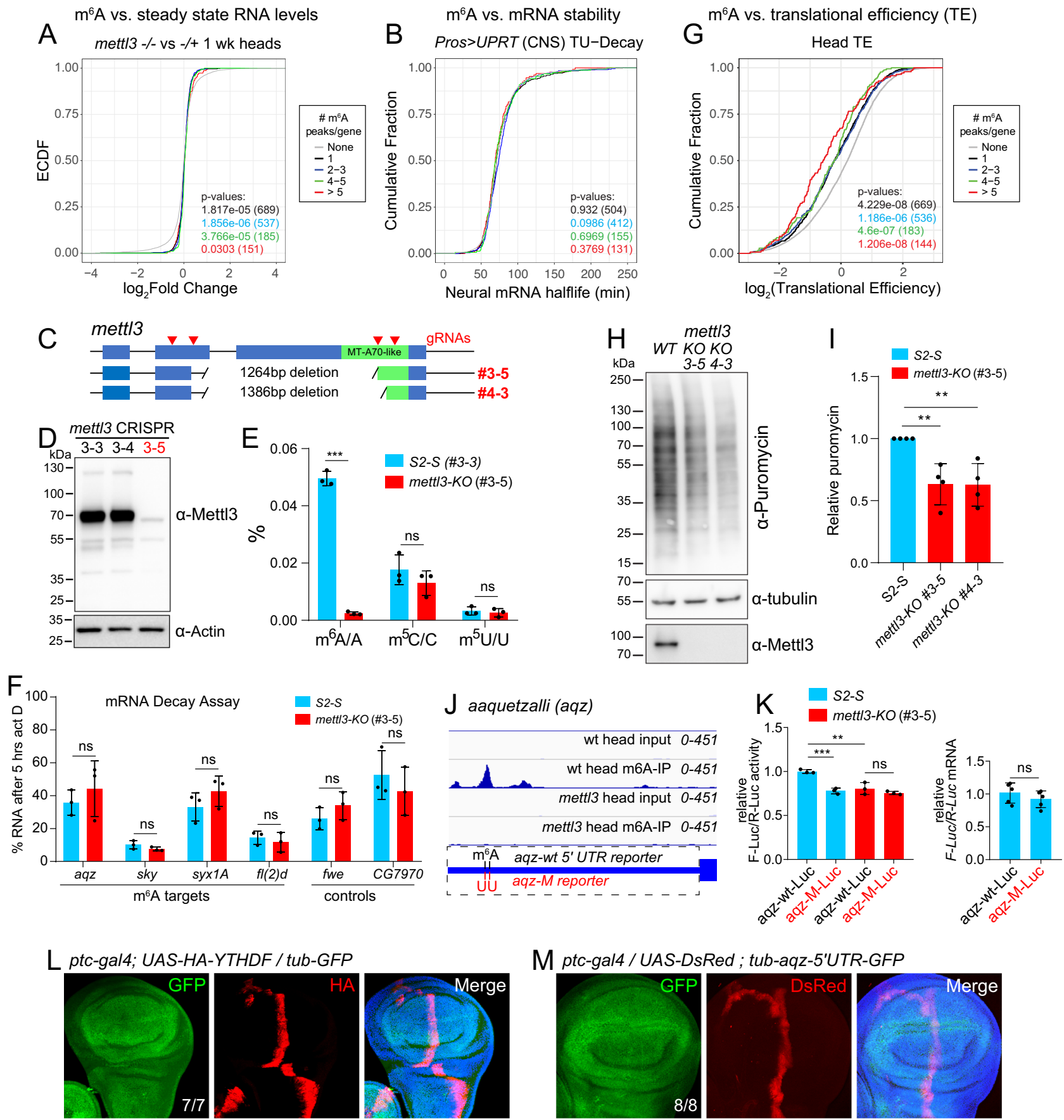

N ptc-gal4; UAS-HA-YTHDF/tub-aqz-5'UTR-GFP
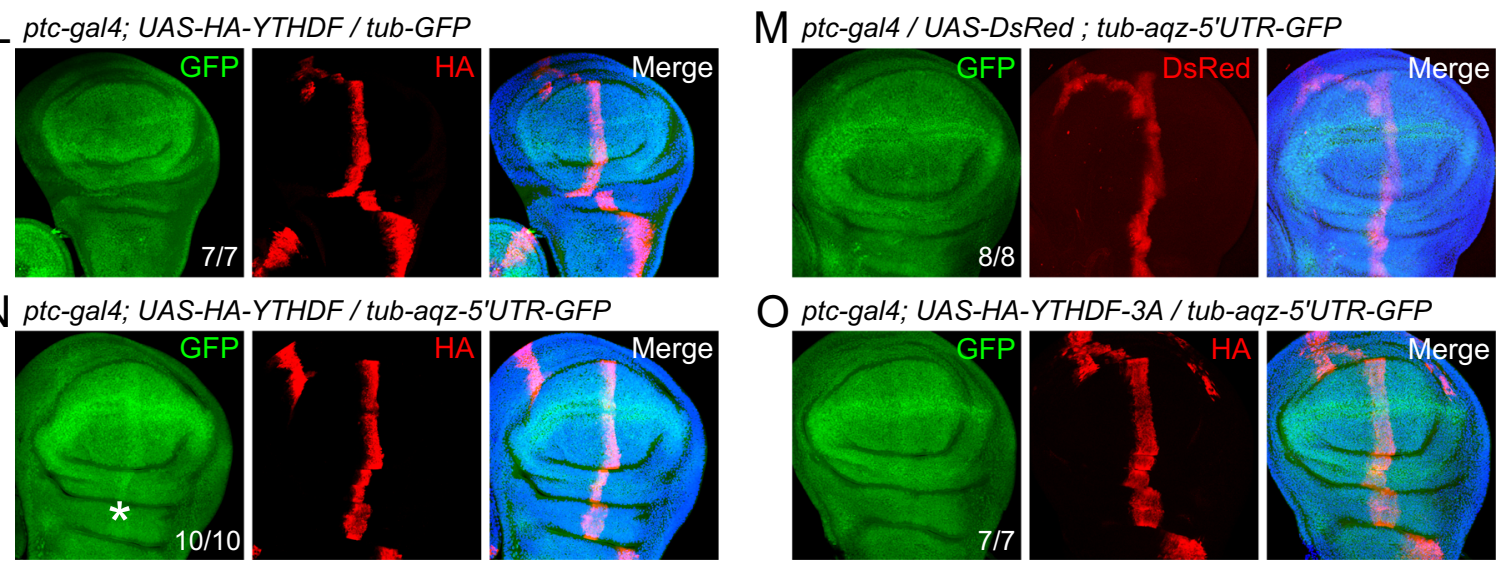

In contrast to prior association of Drosophila $\mathrm{m}^{6} \mathrm{~A}$ with increased steady-state levels of target ${ }^{54}$, we did not observe many changes in our high-confidence $\mathrm{m}^{6} \mathrm{~A}$ targets in Mettl3 (Fig. 6A) or Ythdf mutant CNS from any stage (Supplementary Fig. 10e-h). Paradoxically, even though all bins of $\mathrm{m}^{6} \mathrm{~A}$ targets clustered closely with a $\log _{2}$ fold change of 0 , Kolmogorov-Smirnov (KS) tests indicated statistical significance when comparing sets of $\mathrm{m}^{6} \mathrm{~A}$ targets and background. While statistically different, our

analyses failed to detect any directional gene expression changes in methylated transcripts under writer or reader loss (Fig. 6A and Supplementary Fig. 10).

Since it was conceivable that some expression trends were masked in steady-state measurements, we examined a published dataset of in vivo mRNA decay rates generated using dynamic TU-tagging from the Drosophila CNS, obtained by pulse-chase labeling of pros-Gal4 > UAS-UPRT cells with 4-thiouridine ${ }^{69}$. We 
Fig. $6 \mathbf{m}^{6} \mathbf{A}$ mediates translational activation in Drosophila. A Steady-state RNA levels of $\mathrm{m}^{6} \mathrm{~A}$ targets are not affected by loss of $\mathrm{m}^{6} \mathrm{~A}$. Differential gene expression analysis of 1-week-old Mett/3 heterozygote and mutant heads. No directional change was observed in any group of $\mathrm{m}^{6} \mathrm{~A}$ targets, binned by increasing numbers of Mettl3-dependent $\mathrm{m}^{6} \mathrm{~A}$ peaks. $\mathbf{B} \mathrm{m}^{6} \mathrm{~A}$ targets are not biased in their mRNA stability. The half-life of neural genes obtained from TUdecay measurements ${ }^{69}$ is plotted as cumulative distribution grouping target genes as in (A), based on number of peaks per target. C CRISPR/Cas 9 mutagenesis of Mett/3 in S2-S cells. D Western blotting shows lines \#3-5 and \#4-3 lack Mett/3 protein; \#3-3 was used as a control line. E Quantitative liquid chromatography-mass spectrometry (LC-MS) and absolute quantification confirms specific lack of $\mathrm{m}^{6} \mathrm{~A}$ in Mett/3-KO cells. Error bars, mean $\pm \mathrm{SD}$; $n=3$ biological replicates, two-tailed $t$-test, ${ }^{\star \star \star} p<0.001 . \mathrm{m}^{6} \mathrm{~A} p=5.87 \mathrm{E}-06 . \mathrm{n} . \mathrm{s}$. $=$ non-significant, $\mathrm{m}^{5} \mathrm{C} p=0.604, \mathrm{~m}^{5} \mathrm{U} p=0.612 . \mathbf{F}$ qPCR of $\mathrm{m}^{6} \mathrm{~A}-$ modified mRNAs shows they have similar half-lives in Mett/3-KO and wild-type cells. Error bars, mean $\pm S D ; n=3$ biological replicates, two-tailed $t$-test, $n s$, not significant. G Translation efficiency (TE) measurements ${ }^{70}$ plotted as cumulative fractions for targets with different numbers of $\mathrm{m}^{6} \mathrm{~A}$ peaks. $\mathrm{m}^{6} \mathrm{~A}$ is preferentially deposited on genes with low TE. For panels A, B, and $\mathbf{G}$, a bootstrap method generated the background distribution (None) using genes that lacked $\mathrm{m}^{6} \mathrm{~A}$ peaks. To generate $p$-values, two-sided Kolmogorov-Smirnov (KS) tests were performed comparing the background distribution and each group of $\mathrm{m}^{6} \mathrm{~A}$ target genes. Number of targets are included in parentheses. $\mathbf{H}$ Puromycin labeling ( $5^{\prime}$ in $5 \mu \mathrm{g} / \mathrm{mL}$ media, 50' recovery) shows reduced global protein synthesis in Mett/3-KO cells. I Quantification of nascent protein synthesis in independent Mett/3-KO S2-S cells. Error bars, mean \pm SD; $n=4$ biological replicates, Two-tailed $t$-test, ${ }^{\star \star} p<0.01$. \#3-5 $p=0.00441, \# 4-3 p=0.00496$. J aqz, a model $\mathrm{m}^{6} \mathrm{~A}$ target. We assayed a wildtype aqz $5^{\prime}$ UTR reporter and a variant with two point mutations of $\mathrm{m}^{6} \mathrm{~A}$ sites within the strongest Mettl3-dependent peak (aqz-M). K aqz-wt-Luc generates more reporter output than aqz-M-Luc in wildtype cells, but these have equivalent output in Mett/3-KO cells. Error bars, mean $\pm S D ; n=3$ replicates (luciferase), $n=5$ replicates (qPCR). Two-tailed $t$-test, ${ }^{\star \star} p<0.01,{ }^{\star \star \star} p<0.001 . p=0.0006, p=0.0089$. n.s. non-significant, F-Luc/R-Luc $p=0.278$, mRNA F-Luc/R-Luc $p=$ 0.326. This is one representative result from three independent repeats. L-O Wing imaginal disks expressing tub-GFP (in green), ptc-Gal4 > UAS-HA-Ythdf wild type or $3 A$ mutants or UAS-DsRed (HA/DsRed in red) and DAPI (blue). L Ectopic Ythdf has only marginal effects on the parental tub-GFP reporter. $\mathbf{M}$ DsRed does not affect the tub-aqz-5'UTR-GFP reporter. N-O Wildtype Ythdf ( $\mathbf{N}$ ) but not Ythdf-3A ( $\mathbf{O}$ ) enhances GFP production from tub-aqz-5'UTRGFP. Numbers of disks analyzed are labeled, and representative results are shown. Scale bar is $100 \mu M$. Source data are provided as a Source Data file.

observed that, in aggregate, $\mathrm{m}^{6} \mathrm{~A}$-modified transcripts had identical mRNA half-lives as the background distribution (Fig. 6B). Thus, we were not able to discern global $\mathrm{m}^{6} \mathrm{~A}$ regulatory impacts on transcript properties.

To test this further, we used CRISPR/Cas9 to delete the Mettl3 locus from S2-S cells. Western blotting validated absence of Mettl3 protein in multiple independent cell lines (Supplementary Fig. 11), including two lines that we used for further analysis (\#35 and \#4-3, Fig. 6C, D). Because \#4-3 retained genomic material internal to the confirmed deletion, despite absence of Mettl3 protein (Supplementary Fig. 11), we generally relied on line \#3-5 (Mettl3-KO). Moreover, we directly measured $N^{6}-\mathrm{m}^{6} \mathrm{~A}$ levels in Mettl3-KO cells using quantitative liquid chromatography-mass spectrometry (LC-MS). External calibration curves prepared with $\mathrm{A}$ and $\mathrm{m}^{6} \mathrm{~A}$ standards determined the absolute quantities of each ribonucleoside. The mRNA $\mathrm{m}^{6} \mathrm{~A}$ methylation levels in knockout cells were $<5 \%$ of those in wild-type cells, whereas other modified ribonucleosides were unaffected (Fig. 6E).

Using Mettl3-KO cells, we performed RNA decay assays of validated $\mathrm{m}^{6} \mathrm{~A}$ targets and control transcripts. Following inhibition of transcription using actinomycin $\mathrm{D}$, we observed a range of transcript levels across different loci, but none of these were significantly different between wild-type and $\mathrm{m}^{6} \mathrm{~A}$-deficient cells (Fig. 6F). Overall, our analyses using S2 cells and intact nervous system indicate that mRNA stability of $\mathrm{m}^{6} \mathrm{~A}$-containing transcripts is neither substantially nor directionally influenced by loss of $\mathrm{m}^{6} \mathrm{~A}$ in Drosophila, in contrast to $\mathrm{m}^{6} \mathrm{~A}$ in mammals.

$\mathrm{m}^{6} \mathrm{~A}$ is preferentially deposited on fly transcripts with lower translational efficiency. In light of these data, we examined the alternate possibility of $\mathrm{m}^{6} \mathrm{~A}$-dependent translational control. For this purpose, we utilized ribosome-profiling datasets from Drosophila heads ${ }^{70}$ to assess translational efficiencies of transcripts with or without $\mathrm{m}^{6} \mathrm{~A}$ modifications. Strikingly, we found that genes with $\mathrm{m}^{6} \mathrm{~A}$ had lower translational efficiency than the background distribution (Fig. 6G). The functional relevance of this observation was strengthened by the fact that the number of $\mathrm{m}^{6} \mathrm{~A}$ peaks per transcript exhibited a progressive, inverse correlation with translational efficiency and contrasted with the lack of correlation of $\mathrm{m}^{6} \mathrm{~A}$ modification with either steady-state transcript levels or RNA stability. Altogether, these results suggest that $\mathrm{m}^{6} \mathrm{~A}$ mediates translational control. Moreover, as our miCLIP maps were generated from highly dT-selected RNAs, we infer that this may reflect modifications that are mostly present in cytoplasmic transcripts available for binding to Ythdf.

Drosophila $\mathbf{m}^{6} \mathrm{~A}$ enhances target protein output. The dominant location of Drosophila $\mathrm{m}^{6} \mathrm{~A}$ in $5^{\prime}$ UTRs, contrasting with the preferred residence of mammalian $\mathrm{m}^{6} \mathrm{~A}$ in $3^{\prime} \mathrm{UTR}$, is suggestive of a role in influencing translation. However, the above genomic analyses are correlational in nature, and do not directly connect $\mathrm{m}^{6} \mathrm{~A}$ to gene regulation. One scenario is that $\mathrm{m}^{6} \mathrm{~A}$, being enriched amongst poorly translated mRNAs, is a suppressive mark. However, an alternative logic is that $\mathrm{m}^{6} \mathrm{~A}$ is a positive mark that is preferentially deposited on transcripts that are inefficiently translated, which might make the potential impact of enhancement more overt. Such logic was proposed for mammalian YTHDC2 to enhance translation of low efficiency translated $\mathrm{m}^{6} \mathrm{~A}$ targets $^{38}$.

We first evaluated if $\mathrm{m}^{6} \mathrm{~A}$ might exert global impact on translation. We exploited our Mettl3-KO S2-S cells and monitored newly synthesized proteins using puromycin incorporation ${ }^{71}$. Interestingly, we observe a difference between steadystate protein accumulation and nascent protein synthesis in wildtype and $\mathrm{m}^{6} \mathrm{~A}$-mutant cells. In particular, when analyzing similar amounts of total cellular protein, we observed that both Mettl3KO cells (\#3-5 and \#4-3) consistently generated less newly synthesized bulk proteins than did control S2-S cells (Fig. 6H, I). Western blotting for tubulin, a non- $\mathrm{m}^{6} \mathrm{~A}$ target, verified similar steady-state accumulation between wild-type and knockout cells, while Mettl3 blotting confirmed knockout cell status. These data suggested that $\mathrm{m}^{6} \mathrm{~A}$ may enhance protein output.

We tested this further using a reporter assay. One of the most prominent $\mathrm{m}^{6} \mathrm{~A}$ targets was aaquetzalli (aqz), whose $5^{\prime}$ UTR bore highly Mettl3-dependent miCLIP peaks with multiple CIMS (Fig. 6J), and whose modification we had validated (Fig. 5). Aqz is required for cell polarity and neural development ${ }^{72}$. We cloned its $5^{\prime}$ UTR upstream of firefly luciferase $(a q z-w t-L u c)$, as well as a companion mutant version in which we mutated both identified $\mathrm{m}^{6} \mathrm{~A}$ sites within the strongest miCLIP peak $(a q z-M-L u c)$. We cotransfected these reporters with renilla control reporter into S2-S cells, and observed that the wildtype aqz reporter reliably yielded 
higher output (Fig. 6K). However, when we repeated these tests in Mettl3-KO cells, the wildtype and mutant aqz reporters were indistinguishable (Fig. 6K). Finally, we tested the transcript levels of the reporters by qPCR. While these measurements were more variable than the luciferase activity readouts, they were not significantly different between wt and mutant $a q z$ reporters (Fig. 6K). These tests provide evidence that individual $5^{\prime}$ UTR $\mathrm{m}^{6} \mathrm{~A}$ sites can confer activation in fly cells, and are consistent with translational regulation.

Drosophila Ythdf enhances output of an $\mathrm{m}^{6} \mathrm{~A}$ reporter in a YTH-dependent manner. To test if Ythdf might be an effector of $\mathrm{m}^{6} \mathrm{~A}$-mediated target activation, we implemented a transgenic assay. We used a reporter backbone consisting of GFP under control of the tubulin promoter $(t u b-G F P)$, a transgene that is broadly and relatively evenly expressed in the animal ${ }^{73}$. In this genetic background, we can coexpress factors in a spatially defined subpattern, to assess regulatory impact on the transgene. When we stain wing imaginal disks bearing a naive reporter, and expressing UAS-HA-Ythdf along the anterior-posterior boundary (using ptc-Gal4), we do not observe substantially different GFP protein accumulation in cells co-expressing Ythdf, compared to non-Gal4 cells as internal control territories (Fig. 6L).

Since we had validated $a q z$ as both an $\mathrm{m}^{6} \mathrm{~A}$ target (Fig. $6 \mathrm{~K}$ ) and a Ythdf target (Fig. 5), we transferred its $5^{\prime}$ UTR into the $t u b$-GFP

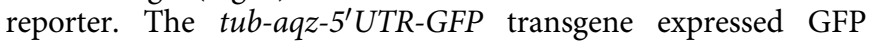
broadly and the levels were not noticeably different from the parent transgene. Its accumulation was not affected by coexpression of a UAS-DsRed transgene (Fig. 6M). However, when we introduced into the $p t c>H A-Y$ thdf background, GFP was elevated specifically within the Ythdf-expressing domain (Fig. 6N). This was consistent with a role for Ythdf in enhancement of this $\mathrm{m}^{6} \mathrm{~A}$ target.

To test if this was due to specific activity of Ythdf, we generated an HA-tagged transgene containing the three YTH pocket mutations, which we showed abrogates association to $\mathrm{m}^{6} \mathrm{~A}$ in vitro (Fig. $1 \mathrm{C}$ ) and to validate $\mathrm{m}^{6} \mathrm{~A}$-bearing transcripts in cells (Fig. 5C). HA-Ythdf-3A protein accumulated to a similar level as wild type, and was also similarly neutral as its wild-type counterpart when tested on the parent tub-GFP reporter. Moreover, mutant Ythdf-3A was unable to enhance GFP protein output from the tub-aqz-5'UTR-GFP transgenic reporter (Fig. 6O). These data support the notion that Ythdf recognizes $\mathrm{m}^{6} \mathrm{~A}$-bearing $5^{\prime} \mathrm{UTR}$ targets for translational enhancement.

An autonomous, $\mathrm{m}^{\mathbf{6}} \mathrm{A}$-dependent, neural function for Ythdf in memory. The availability of wild-type and mutant Ythdf transgenes allowed us to conduct further genetic tests of the connection between $\mathrm{m}^{6} \mathrm{~A}$ readout and STM. Overexpression of either Ythdf transgene, (e.g. with ptc-Gal4, ap-Gal4) did not substantially impair viability or developmental patterning; and pan-neuronal expression of Ythdf using elav-Gal4 did not affect lifespan (Supplementary Fig. 4). Therefore, even though we could detect a selective $\mathrm{m}^{6} \mathrm{~A}$-dependent impact of Ythdf on reporter transgenes, elevated Ythdf expression does not interfere with normal developmental programs, or has effects that are otherwise within the range of developmental compensation. This mirrors the lack of substantial consequences of removing Ythdf.

We moved to perform cell-type-specific transgenic rescue assays. Building on our observation that neural-knockdown of Ythdf phenocopied the STM defects seen in whole-animal mutations (Fig. 3G), we introduced elav-Gal4 and UAS-Ythdf$w t$ or UAS-Ythdf-3A transgenes into Ythdf hemizygous null backgrounds. The Ythdf mutants carrying elav-Gal4 had defective STM, indicating the Gal4 transgene does not improve this behavioral output (Fig. 7A). This was important to rule out, since at least some other Drosophila neuronal phenotypes are modified by Gal4 alone ${ }^{74,75}$. With this control background as reference, we found that the STM deficit of Ythdf nulls could be rescued by pan-neuronal restoration of Ythdf, thereby restoring normal STM (Fig. 7A). In contrast, elav $>$ Ythdf-3A transgenes did not rescue normal STM capacity to Ythdf mutants (Fig. 7A). Thus, $\mathrm{m}^{6} \mathrm{~A}$ binding is critical for neural Ythdf function during STM formation.

An $m^{6} \mathrm{~A} /$ Ythdf pathway functions specifically in $\mathrm{MB}$ to promote memory. In Drosophila, associative olfactory memory is stored in the intrinsic neurons of the $\mathrm{MB}^{9,76}$. Bearing in mind that Ythdf-mediated regulation has particular impact on STM, we evaluated the effects of its gain-of-function on wild-type $\mathrm{MB}$ neurons using MB247-Gal4. Strikingly, MB247-Gal4 > Ythdf flies exhibited compromised STM formation at 20 days, while MB247Gal4 $>$ Ythdf-3A flies were normal (Fig. 7B). This behavioral defect was relatively specific: flies overexpressing wild-type and mutant Ythdf exhibited similar locomotor activity when quantified over $60 \mathrm{~s}$ of tracking (Fig. 7C). Thus, ectopic Ythdf disrupts STM in an $\mathrm{m}^{6} \mathrm{~A}$-dependent manner.

These data prompted us to investigate the endogenous $\mathrm{m}^{6} \mathrm{~A} /$ Ythdf pathway with respect to the MB more rigorously. Flies depleted of Mettl3 in the MB exhibited STM impairment (Fig. 7D), comparable to whole-animal Mettl3 null mutants (Fig. 2). Similarly, MB247-Gal4 > UAS-Ythdf[RNAi] flies exhibited substantial STM defects (Fig. 7E), comparable to Ythdf null animals (Fig. 3F, G). Thus, Mettl3 and Ythdf are specifically required in $\mathrm{MB}$ neurons to facilitate normal STM.

It was possible that these $\mathrm{m}^{6} \mathrm{~A}$ factors act not only in $\mathrm{MB}$ neurons, but also in other neural populations, to promote STM. To address the sufficiency of the $\mathrm{m}^{6} \mathrm{~A} / \mathrm{Y}$ thdf pathway in $\mathrm{MB}$ neurons, we conducted further transgenic rescue tests. Recall that we were able to rescue Mettl3 null animals (Fig. 2G) and Ythdf null animals (Fig. 7A) by pan-neuronal expression of these factors. Now, we tested whether we could do so by expressing them only using MB247-Gal4. Indeed, this fully rescued the STM defects of Mettl3 mutants (Fig. 7F) and substantially improved those of Ythdf mutants (Fig. 7G). Therefore, even though $\mathrm{m}^{6} \mathrm{~A}$ regulation undoubtedly impacts most cell types in the animal, the $\mathrm{m}^{6} \mathrm{~A} / \mathrm{Y}$ thdf pathway plays a cell-autonomous role in $\mathrm{MB}$ neurons to mediate STM.

To investigate whether this might be potentially associated with effects on MB structure, we introduced a UAS-GFP transgene into the MB247-Gal4, Mettl3[null] mutant background, which facilitated visualization of the mushroom-body lobes. In Mettl3 heterozyotes, the $\mathrm{MB}$ adopts its characteristic morphology: the left and right $\mathrm{MB}$ horizontal lobes are distinct (Fig. $7 \mathrm{H}$, top left). However, a majority of Mettl3 mutants exhibit fusion of the MB $\beta$-lobes (Fig. $7 \mathrm{H}$, top right). We did not observe comparable fusion of the $\gamma$-lobes or shortening of the $\alpha$-lobes, indicating a spatially restricted effect. We initially conducted these tests at 3 weeks, to align with our functional tests of learning and memory. However, preferential fusion of Mettl3 mutant MB $\beta$ lobes was also detected at 1 week, even though their behavioral performance was normal at this time (Supplementary Fig. 3). Accordingly, we tested Ythdf heterozygous and hemizygous mutants, and similarly observed qualitatively similar fusion of MB $\beta$-lobes in mutants (Fig. 7I), at both 1 and 3 weeks. Our MB staining data of the two mutants at both timepoints are quantified in Fig. 7J. 

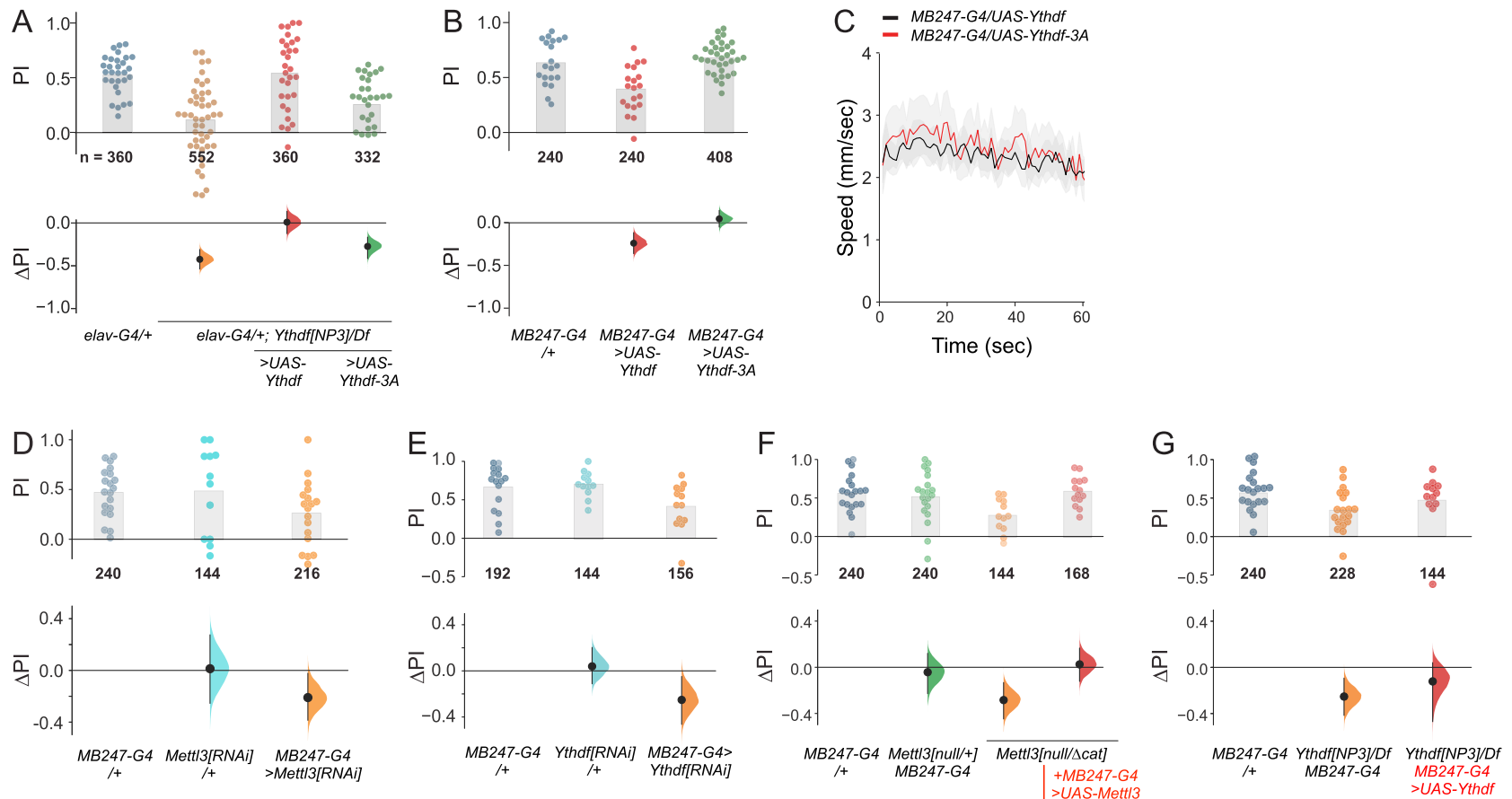

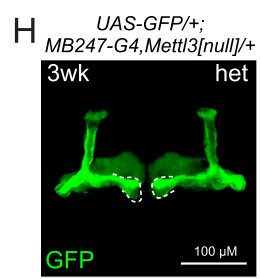

no fusion
$U A S-G F P /+$

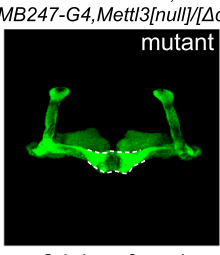

$\beta$-lobes fused

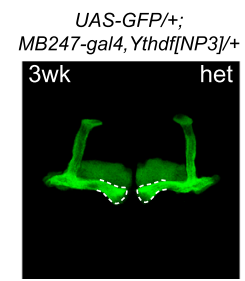

no fusion
UAS-GFP/+;

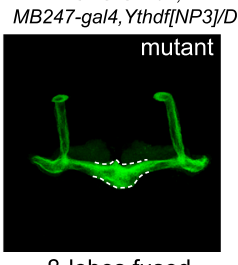

$\beta$-lobes fused

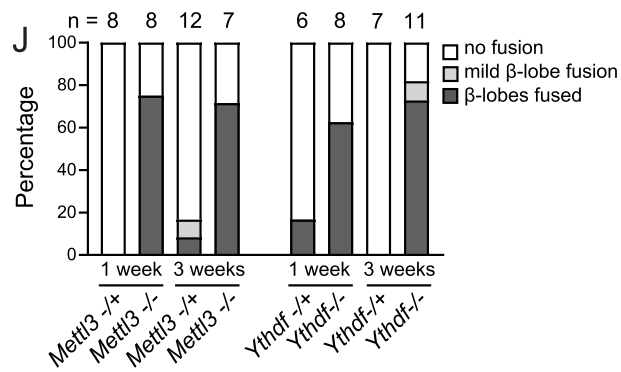

Fig. 7 An $\mathbf{~ m}^{6} \mathbf{A} /$ Ythdf pathway acts in the mushroom body to mediate STM. A-C In vivo function of Ythdf during learning and memory requires $m^{6} A$ binding capacity. A Pan-neuronal expression (using elav-Gal4, =G4) of wild-type Ythdf, but not Ythdf-3A, rescued STM defects in Ythdf hemizygote mutants at 20 days. Elav-G4; Ythdf[NP3]/Df vs. elav-G4/ $+=-0.42[95 \mathrm{Cl}-0.53,-0.31] p<1^{\star} 10^{-4}$. Elav-G4; UAS-Df1; Ythdf[NP3]/Df vs. elav-G4/+ =0 $[95 \mathrm{Cl}-0.13,+0.14] p=0.684$. Elav-G4; UAS-Df1-3A; Ythdf[NP3]/Df vs elav-G4/+ $=-0.27[95 \mathrm{Cl}-0.41,-0.17] p<1^{\star} 10^{-4}$. B Mushroom body (MB) expression (using MB247-Gal4) of Ythdf, but not Ythdf-3A, impairs STM in 20-day flies. MB247-G4; UAS-Ythdf $-0.24[95 \mathrm{Cl}-0.37,-0.12] p=0.001$. MB247-G4/+ vs. MB247-G4; UAS-Ythdf-3A (vs. MB247-G4/+ $=+0.04[95 \mathrm{Cl}-0.05,+0.15] p=0.415$. C Expression of Ythdf-wt/3A proteins does not affect locomotion behavior. D Depletion of Mett/3 in the MB phenocopied STM impairment seen in whole animal Mett/3 mutants (see Fig. 2). Mett/3 $[R N A i] /+$ vs. MB247-G4/+ = +0.01[95Cl -0.26, +0.27] $p=0.654$. MB247-G4; UAS-Mett/3[RNAi] vs. MB247-G4/ $+=-0.21[95 \mathrm{Cl}-0.38,-0.02] p=$ 0.034. E MB-specific knockdown of Ythdf compromised STM, similar to what has been observed in whole animal Ythdf mutants (see Fig. 3). Ythdf[RNAi]/+ vs. MB247-G4/+=+0.04[95Cl $-0.11,+0.2] p=0.835$. MB247-G4; UAS-Ythdf[RNAi] vs MB247-G4/ $+-0.25[95 \mathrm{Cl}-0.46,-0.05] p=0.002$. F Reexpression of Mett/3 only in MB neurons rescued STM defects in Mett/3 trans-heterozygous mutants. MB247-G4; UAS-Mett/3[null]/+ vs. MB247-G4/+ $-0.04[95 \mathrm{Cl}-0.23,+0.12] p=0.86$. Mett/3[null]/Mett/3[Acat] vs. MB247-G4/+ = $-0.28[95 \mathrm{Cl}-0.45,-0.13] p=0.004$. MB247-G4/UAS-Mett/3;

Mett/3[null] $/$ Mett/3[Acat] vs. MB247-G4/ $+=+0.02[95 \mathrm{Cl}-0.12,+0.17] p=0.766$. G Re-expression of Ythdf only in MB neurons improves STM defects in Ythdf hemizygous mutants. MB247-G4; Ythdf[NP3]/Df vs. MB247-G4/+ = -0.25[95Cl -0.41, -0.09] $p=0.005$. MB247-G4/UAS-Ythdf; Ythdf[NP3]/Df vs. MB247-G4/+ = $-0.12[95 \mathrm{Cl}-0.47,+0.03] p=0.572$. STM and locomotion assays were conducted in 20-day-old flies. Each dot in the STM scatter plots represents a PI average of 12 flies. All control-test differences are displayed as effect sizes with error curves and $95 \%$ confidence intervals. No nullhypothesis significance testing was performed; two-tailed Mann-Whitney $P$ values are shown for legacy purposes only. For multiple comparisons, several test groups were compared against a common control group. $\mathbf{H}$ and I MB structural defects in Mett/3 (H) and Ythdf (I) mutants at 3 weeks. All panels depict GFP driven by MB247-Gal4. H Representative Mett/3 heterozygote (left) and trans-heterozygote mutant (right), showing fusion of $\beta$-lobes in the latter. I Representative Ythdf heterozygote (left) and hemizygote mutant (right), showing fusion of $\beta$-lobes in the latter. J Quantification of genotypes analyzed in $\mathbf{H}$ and $\mathbf{I}$ at 1 and 3 weeks; $n$ sizes are labeled. Source data are provided as a Source Data file.

At this point, it remains to be determined whether the $\mathrm{MB}$ structural defects are diagnostic and a more sensitive readout that precedes subsequent behavioral deficits, or whether these phenotypes occur in parallel. In either scenario, these tests provide substantial evidence that a Drosophila $\mathrm{m}^{6} \mathrm{~A} / \mathrm{Ythdf}$ pathway maintains $\mathrm{MB}$ structure, and operates cell-autonomously in the $\mathrm{MB}$ to mediate odor-avoidance learning.

\section{Discussion}

Distinct local contexts, genic location, and regulatory impact for $\mathbf{m}^{6} \mathbf{A}$ in different metazoans. Despite tremendous interests in the regulatory utilities and biological impacts of mRNA methylation, there has been relatively little study from invertebrate models. Given that the $\mathrm{m}^{6} \mathrm{~A}$ pathway seems to have been lost from C. elegans, Drosophila is an ideal choice for this. Since the 
initial report that Mettl3 mutants affect germline development ${ }^{52}$, we and others showed that Drosophila harbors an $\mathrm{m}^{6} \mathrm{~A}$ pathway similar to that of mammals, but simplified in that it has a single nuclear and cytoplasmic YTH reader ${ }^{53-55}$. Nevertheless, Drosophila has proven to be a useful system to discover and characterize novel $\mathrm{m}^{6} \mathrm{~A}$ factors ${ }^{54,55,77,78}$. Expanding the breadth of model systems can increase our appreciation for the utilization and impact of this regulatory modification.

It is widely presumed, based on mammalian profiling, that metazoan $\mathrm{m}^{6} \mathrm{~A}$ is enriched at stop codons and $3^{\prime}$ UTRs. However, our high-resolution maps indicate that $5^{\prime}$ UTRs are by far the dominant location of methylation in mature Drosophila mRNAs. Although further study is required, many of these $\mathrm{m}^{6} \mathrm{~A} 5^{\prime} \mathrm{UTR}$ regions coincide with our previous embryo miCLIP data (e.g. Supplementary Fig. 7), while other miCLIP CIMs calls located in other transcript regions ${ }^{55}$ proved usually not to be Mettl3dependent. Thus, our data indicate a fundamentally different distribution of $\mathrm{m}^{6} \mathrm{~A}$ in Drosophila mRNAs compared to mammals.

While mammalian $\mathrm{m}^{6} \mathrm{~A}$ clearly elicits a diversity of regulatory consequences, depending on genic and cellular context and other factors, a dominant role is to induce target decay through one or more cytoplasmic YTH readers. This harkens back to classic observations that $\mathrm{m}^{6} \mathrm{~A}$ is correlated with preferential transcript decay $^{79}$, and more recent data that loss of $\mathrm{m}^{6} \mathrm{~A}$ writers ${ }^{80-82}$ or cytoplasmic YTH readers ${ }^{29,32}$ results in directional upregulation of $\mathrm{m}^{6} \mathrm{~A}$ targets. However, several lines of study did not yield convincing evidence for a broad role for the Drosophila $\mathrm{m}^{6} \mathrm{~A}$ pathway in target decay. Instead, the dominant localization of $\mathrm{m}^{6} \mathrm{~A}$ in fly $5^{\prime}$ UTRs is suggestive of a possible impact in translational regulation. Our genomic and genetic evidence support the notion that $\mathrm{m}^{6} \mathrm{~A}$ is preferentially deposited in transcripts with overall lower translational efficiency, but that $\mathrm{m}^{6} \mathrm{~A} / \mathrm{Ythdf}$ may potentiate translation. However, we can rationalize a regulatory basis for these apparently opposite trends, if the greater modulatory window of poorly translated loci is utilized for preferred targeting by $\mathrm{m}^{6} \mathrm{~A} / \mathrm{Y}$ thdf.

As is generally the case for mammalian $\mathrm{m}^{6} \mathrm{~A}$, the choice of how appropriate targets are selected for modification, and which gene regions are preferentially methylated, remains to be understood. The minimal context for $\mathrm{m}^{6} \mathrm{~A}$ is insufficient to explain targeting, and as mentioned also seems to be different between Drosophila and vertebrates. A further challenge for the future will be to elucidate a mechanism for $\mathrm{m}^{6} \mathrm{~A} /$ Ythdf-mediated translational regulation. This will reveal possible similarities or distinctions with the multiple strategies proposed for translational regulation by mammalian $\mathrm{m}^{6} \mathrm{~A}$, which include both cap-independent translation via $5^{\prime}$ UTRs during the heat-shock response via eIF $3^{83}$ or YTHDF2 $^{36}$; cap-dependent mRNA circularization via Mettl3-eIF3 $\mathrm{H}^{84}$; and activity-dependent translational activation in neurons ${ }^{34}$.

Roles for the $\mathrm{m}^{6} \mathrm{~A} / \mathrm{DF} 1$ pathway in learning and memory. Recent studies have highlighted neuronal functions of mammalian $\mathrm{m}^{6} \mathrm{~A}$ pathway factors ${ }^{39}$. There is a growing appreciation that mouse mutants of multiple components in the $\mathrm{m}^{6} \mathrm{~A}$ RNAmodification machinery affect learning and memory $34,46,48-50$. Here, we provide substantial evidence that, in Drosophila, neural $\mathrm{m}^{6} \mathrm{~A}$ is critical for STM. We specifically focused on STM as this paradigm has been extensively characterized in Drosophila. Mouse studies have almost exclusively examined effects on LTM, and these two memory phases are mechanistically distinct ${ }^{85,86}$. One main distinction is that LTM requires protein synthesis after training, while STM does not. So, while direct comparisons between the two systems are not possible, it is nevertheless instructive to consider the parallels and distinctions of how $\mathrm{m}^{6} \mathrm{~A}$ facilitates normal memory function in these species. This is especially relevant given that both mouse and fly central nervous systems require a cytoplasmic YTH factor for memory.

In mice, the $\mathrm{m}^{6} \mathrm{~A}$ writer Mettl3 enhance long-term memory consolidation, potentially by promoting the expression of genes such as $A r c, c$-Fos and others ${ }^{46}$. Another study found that Mettl14 is required for LTM formation and neuronal excitability 49 . Conversely, knockdown of the $\mathrm{m}^{6} \mathrm{~A}$ demethylase FTO in the mouse prefrontal cortex resulted in enhanced memory consolidation $^{48}$. Amongst mammalian YTH $\mathrm{m}^{6} \mathrm{~A}$ readers, YTHDF1 was shown to induce the translation of $\mathrm{m}^{6} \mathrm{~A}$-marked mRNA specifically in stimulated neurons ${ }^{34}$. In cultured hippocampal neurons, levels of YTHDF1 in the PSD fraction were found to increase by $\sim 30 \%$ following $\mathrm{KCl}$ treatment. This suggests that YTHDF1 concentration at the synapse could be critical for regulating the expression levels of proteins (such as CaMK2a) involved in synaptic plasticity ${ }^{87}$. Taken together, these studies suggest the $\mathrm{m}^{6} \mathrm{~A}$ pathway is a crucial mechanism of LTM consolidation in mammals that optimizes animal behavioral responses.

Of note, the genetics and sample sizes possible in Drosophila permit comprehensive, stringent, and anatomically resolved analyses ${ }^{88}$. Thus, in our study, we systematically analyze all writer and reader factors, and reveal a notable functional segregation, suggesting that the cytoplasmic reader Ythdf is a major effector of Mettl3/Mettl14 $\mathrm{m}^{6} \mathrm{~A}$ in memory. Given that Ythdf mutants otherwise exhibit few overt developmental or behavioral defects in normal or sensitized backgrounds (while Ythdc1 mutants generally phenocopy Mettl3/Mettl14 mutants) its role in STM is a surprising insight into the contribution of Ythdf to a critical adaptive function. Moreover, we can pinpoint the spatial requirements of $\mathrm{m}^{6} \mathrm{~A}$ for STM, by showing that (1) neuronal-specific and MB-specific depletion of Mettl3/Ythdf can induce defective STM, and (2) neuronal and MB-specific restoration of Mettl3 or Ythdf to their respective whole-animal knockouts restores normal STM. Moreover, the fact that Ythdf gain-of-function in the MB can also disrupt STM, but does not generally alter other aspects of development or behavior, points to a homeostatic role of $\mathrm{m}^{6} \mathrm{~A}$ regulation in Drosophila learning and memory.

We observed that STM defects in fly $\mathrm{m}^{6} \mathrm{~A}$ mutants are agedependent, which has not been reported in mammals. Although many physiological capacities decline with life history, the observed STM defects seem to be decoupled from other agerelated phenotypes, since mutation of Ythdf or neural overexpression of Ythdf can interfere with STM but does not substantially impact lifespan or locomotion. In this regard, Mettl3 and Ythdf are different from classical memory genes such as rutabaga ${ }^{89}$ because STM impairment in $\mathrm{m}^{6} \mathrm{~A}$ mutants was absent in young flies and only became apparent with progressing age.

One interpretation is that there is a cumulative effect of deregulated $\mathrm{m}^{6} \mathrm{~A}$ networks that has a progressive impact specific to mushroom-body neurons. To gain further mechanistic insights, future studies will need to examine age-related changes in gene expression and/or translation, in a cell-specific manner. It remains to be seen whether specific deregulated targets downstream of Ythdf have large individual effects, or whether the STM deficits arise from myriad small effects on translation. YthdfCLIP and ribosome profiling from the CNS may prove useful to decipher this. Assuming that loss of translational enhancement of $\mathrm{m}^{6} \mathrm{~A} /$ Ythdf targets mediates STM defects, one possibility, to be explored in future studies, is that some targets may already be known from prior genetic studies of memory ${ }^{5}$. 


\section{Methods}

$\mathbf{m}^{\mathbf{6}} \mathbf{A}$ reader constructs. We obtained full-length cDNAs obtained by PCR from a cDNA library for Ythdc1 (encoding Ythdc1-PA, 721aa), and Ythdf (encoding Ythdf-PA, 700aa) into pENTR vector. We then used site-directed mutagenesis (primers listed in Supplementary Dataset 6) to generate pENTR-Ythdc1-3A (w276A w327A L338A), and pENTR-Ythdf-3A (w404A-W459A-w464A). These were transferred into the Drosophila Gateway vector pAGW (N-terminal GFP fusion) and pAHW (N-terminal HA tag) to make all combinations of tagged wildtype and " $3 \mathrm{~A}$ " mutant versions for expression in Drosophila cell culture. We also cloned the Ythdf sequences into pTHW (N-terminal HA fusion) to generate UASHA-Ythdf and UAS-HA-Ythdf-3A for transgenes. For mammalian expression, we cloned wild-type and " $3 A$ " mutant versions of Ythdc1 and Ythdf into pcDNA5/ FRT/TO with an N-terminal 3xFlag-tag.

$\mathbf{m}^{6} \mathbf{A}$ probe pulldown with fly Ythdf and Ythdc1. As we were unable to purify sufficient amounts of full-length Drosophila Ythdf and Ythdcl proteins from Drosophila cell culture for in vitro characterization, we instead turned to purifying them from mammalian cells. For initial interaction tests of wild-type Flag-Ythdc1/ DF with $\mathrm{A} / \mathrm{m}^{6} \mathrm{~A}$ RNA probes, we seeded 4 million HEK293T cells in a $10 \mathrm{~cm}$ dish $24 \mathrm{~h}$ prior to calcium phosphate transfection, and used $10 \mu \mathrm{g}$ of plasmid DNA (pcDNA-3xFlag-Ythdc1/DF constructs) per $10 \mathrm{~cm}$ dish. Cells were harvested $24 \mathrm{~h}$ post transfection. Cells from one $10 \mathrm{~cm}$ dish and $0.6 \mathrm{~mL}$ of lysis buffer were used per condition. Cells were lysed in NP-40 lysis buffer $(50 \mathrm{mM}$ Tris-HCl pH 7.5, $150 \mathrm{mM} \mathrm{NaCl}, 0.5 \% \mathrm{NP}-40,5 \mathrm{mM} \mathrm{MgCl}$, EDTA-free protease inhibitor tablet (Roche), $1 \mathrm{mM}$ PMSF) on ice. Clarified lysate was then incubated with $\mathrm{A}$ or $\mathrm{m}^{6} \mathrm{~A}$ containing RNA probe (Supplementary Dataset 6 ) on ice at $1 \mu \mathrm{M}$ concentration for $20 \mathrm{~min}$. Reactions were then irradiated with $365 \mathrm{~nm}$ UV (Spectroline ML-3500S) on ice for $10 \mathrm{~min}$. The reaction was then incubated with $60 \mu \mathrm{L}$ of high capacity streptavidin agarose $50 \%$ bead slurry (Pierce $\# 20357$ ) at $4{ }^{\circ} \mathrm{C}$ on a rotatory wheel for $3 \mathrm{~h}$. The beads were then washed with $1 \%$ SDS in TBS $(3 \times 1 \mathrm{~mL}), 6 \mathrm{M}$ urea in TBS $(3 \times 1 \mathrm{~mL})$, and TBS $(3 \times 1 \mathrm{~mL})$. The RNA-bound proteins were eluted by boiling the beads in $50 \mu \mathrm{L}$ of $1 \times$ Laemmli sample buffer $(80 \mathrm{mM}$ Tris-HCl pH 6.8, $2 \%$ SDS, $10 \%$ glycerol, $5 \%$ B-mercaptoethanol, $0.02 \%$ bromophenol blue) at $95^{\circ} \mathrm{C}$ for $5 \mathrm{~min}$. The input, flow-through, and eluates were separated by SDS-PAGE and analyzed by Western blotting using mouse $\alpha$-Flag M2 (Sigma \#F1804).

In the experiment where 3A mutants of Ythdf and Ythdc1 were tested, cells from two $10 \mathrm{~cm}$ dishes and $1.2 \mathrm{~mL}$ of lysis buffer were used per condition. The cross-linking and pull-down were performed as described above. AAACU and AA$\mathrm{m}^{6} \mathrm{~A}-\mathrm{CU}$ sequences were used for this experiment.

\section{Proteomic profiling of the fly $\mathrm{m}^{6} \mathrm{~A}$ interactome}

Mass spectrometry analysis. For proteomics experiments with Drosophila S2 cells, we adapted our previously described method ${ }^{57}$. S2 cells were lysed by cryomilling. The resulting cell powder $(750 \mathrm{mg})$ was first extracted with $1.5 \mathrm{~mL}$ of low-salt extraction buffer (20 mM Tris- $\mathrm{HCl} \mathrm{pH} \mathrm{7.5,} 10 \mathrm{mM} \mathrm{NaCl}, 2 \mathrm{mM} \mathrm{MgCl}$, $0.5 \%$ Triton X-100, $10 \%$ glycerol, protease inhibitor tablet (Roche), and phosphatase inhibitor (Pierce)), and then $1 \mathrm{~mL}$ of high-salt extraction buffer $(50 \mathrm{mM}$ Tris- $\mathrm{HCl} \mathrm{pH} \mathrm{7.5,} 420 \mathrm{mM} \mathrm{NaCl}, 2$ $\mathrm{mM} \mathrm{MgCl} 2,0.5 \%$ Triton X-100, 10\% glycerol, protease inhibitor tablet (Sigma) and phosphatase inhibitor (Pierce)). Low-salt and high-salt extracts were pooled, and protein concentration was determined by Bradford assay. The pooled extract was diluted to $3 \mathrm{mg} / \mathrm{mL}$ if needed before proceeding to photo-crosslinking.

AAACU or AA-m ${ }^{6} \mathrm{~A}-\mathrm{CU}$ oligo probe was added to $2 \mathrm{~mL}$ of extract to a final concentration of $1 \mu \mathrm{M}$. The reactions were incubated on ice for $20 \mathrm{~min}$ prior to photo-cross-linking. The reactions were then irradiated with $365 \mathrm{~nm}$ UV (Spectroline ML-3500S) on ice for $15 \mathrm{~min}$. The reaction was then incubated with $60 \mu \mathrm{L}$ of high capacity streptavidin agarose $50 \%$ bead slurry (Pierce \#20357) at $4{ }^{\circ} \mathrm{C}$ on a rotatory wheel for $3 \mathrm{~h}$. The beads were then washed with $1 \%$ SDS in TBS $(3 \times$ $1 \mathrm{~mL}), 6 \mathrm{M}$ urea in TBS $(3 \times 1 \mathrm{~mL})$, and TBS $(3 \times 1 \mathrm{~mL})$. The RNA-bound proteins were eluted with RNase cocktail (Thermo Fisher) in RNase elution buffer $(10 \mathrm{mM}$ Tris- $\mathrm{HCl} \mathrm{pH} \mathrm{7.5,} 40 \mathrm{mM} \mathrm{NaCl}, 1 \mathrm{mM} \mathrm{MgCl}$ ) at $37^{\circ} \mathrm{C}$ for 30 min with periodic agitation.

The proteomics files were searched against Drosophila melanogaster database downloaded from UniProt (https://www.uniprot.org/). To plot the mass spectrometry data (Fig. 1d), we first removed 242 proteins that were not consistently recovered in both replicate datasets, leaving 353 proteins. To calculate enrichment ratios for proteins identified by only one probe, we added 1 to all spectral count values. Proteins that do not exhibit differential binding to the A/ $\mathrm{m}^{6} \mathrm{~A}$ probes cluster around the plot origin, and we thresholded at $2.0 \times$ the interquartile range.

Analysis of $\mathbf{m}^{\mathbf{6}} \mathbf{A}$ by liquid chromatography-coupled mass spectrometry. Total RNA was extracted from Drosophila S2 cells and whole female fly (one-week old) using TRIzol reagent and subjected to DNase treatment (Thermo Fisher \#AM1907). The mRNA was then isolated through two rounds of poly-A selection using the oligo-d(T) ${ }_{25}$ beads (NEB \#S1419S). The RNA was digested with nuclease P1 (Wako USA \#145-08221) and dephosphorylated with Antarctic phosphatase (NEB \#M0289S). Briefly, $1 \mu \mathrm{g}$ of RNA was digested with 2 units of nuclease P1 in buffer containing $7 \mathrm{mM} \mathrm{NaOAc} \mathrm{pH} 5.2,0.4 \mathrm{mM} \mathrm{ZnCl}_{2}$ in a total volume of $30 \mu \mathrm{L}$ at $37^{\circ} \mathrm{C}$ for $2 \mathrm{~h} .3 .5 \mu \mathrm{L}$ of $10 \times$ Antarctic phosphatase buffer and $1.5 \mu \mathrm{L}$ of Antarctic phosphatase was then directly added to the reaction and incubated at $37^{\circ} \mathrm{C}$ for another $2 \mathrm{~h}$.

Quantitative LC-MS analysis of $\mathrm{m}^{6} \mathrm{~A}$ was performed on an Agilent 1260 Infinity II HPLC coupled to an Agilent 6470 triple quadrupole mass spectrometer in positive ion mode using dynamic multiple reaction monitoring (DMRM). The ribonucleosides in the digested RNA samples were separated by a Hypersil GOLD C18 Selectivity HPLC Column (Thermo Fisher \#25003-152130; $3 \mu \mathrm{m}$ particle size, $175 \AA$ pore size, $\left.2.1 \times 150 \mathrm{~mm} ; 36^{\circ} \mathrm{C}\right)$ at $0.4 \mathrm{~mL} / \mathrm{min}$ using a solvent system consisting of $0.1 \%$ formic acid in $\mathrm{H}_{2} \mathrm{O}$ (A) and acetonitrile (B) based upon literature precedent ${ }^{90}$. The operating parameters for the mass spectrometer were as follows: gas temperature $325^{\circ} \mathrm{C}$; gas flow $12 \mathrm{~L} / \mathrm{min}$; nebulizer $20 \mathrm{psi}$ and capillary voltage $2500 \mathrm{~V}$, with fragmentor voltage and collision energy optimized for each different nucleoside. The nucleosides were identified based on the transition of the parent ion to the deglycosylated base ion: $m / z 282 \rightarrow 150$ for $\mathrm{m}^{6} \mathrm{~A}$ and $\mathrm{m} / z 268 \rightarrow$ 136 for A. Calibration curves were constructed for each nucleoside using standards prepared from commercially available ribonucleosides. The level of $\mathrm{m}^{6} \mathrm{~A}$ was determined by normalizing $\mathrm{m}^{6} \mathrm{~A}$ concentration to A concentration in the sample.

Drosophila stocks. Ythdf[NP3], FRT40A and Mettl14[sk1]/Tb-RFP cyo,w+ were previously described ${ }^{55}$. Ythdcl[ $[\Delta N] / D f d-Y F P, T M 3 ;$ Mettl3[Acat]/TM6C, Mettl3 [null]/TM6C, Mettl14[fs]/Tb-RFP cyo,w+; UAS-Mettl3-HA were a gift of Jean-Yves Roignant ${ }^{54}$. All of these were genotyped in trans to deficiencies (see Supplementary Dataset 6) to confirm the absence of the wild-type allele. Other stocks were obtained from the Bloomington Drosophila Stock Center. Deficiency lines: $D f(3 R)$ Exel6197 (BL-41590, removes Mettl3), Df(3L)ED208 (BL-34627, removes Ythdc1), $D f(3 R) B S C 461$ (BL-24965, removes Ythdf) and Df(3R)BSC655 (BL-26507, removes Ythdf). TRiP knockdown lines: Mettl3 (BL-41590), Mettl14 (BL-64547), Ythdc1 (BL-34627) and Ythdf (BL-55151). Gal4 lines: elav-gal4 (BL-8765), elav[C155]-Gal4 (BL-458), ptc-Gal4 (BL-2017), tub-Gal4 (BL-5138), da-Gal4 (BL-55851), ap-Gal4 (BL-3041), MB247-Gal4 (mef2-Gal4, BL-50742).

To generate the tub-aqz-GFP reporter, we cloned the aqz 5'UTR (chr3R:8,818, 731-8,820,682) into the $5^{\prime} \mathrm{UTR}$ position of the tub-GFP vector (KpnI/BamHI). tubaqz-GFP, UAS-HA-Ythdf and UAS-HA-Ythdf-3A (described above) were injected into $w[1118]$ with $\Delta 2-3$ helper plasmid to obtain transformants (Bestgene, Inc.)

Flies were raised on standard cornmeal-based food medium containing $1.25 \%$ $\mathrm{w} / \mathrm{v}$ agar, $10.5 \% \mathrm{w} / \mathrm{v}$ dextrose, $10.5 \% \mathrm{w} / \mathrm{v}$ maize, and $2.1 \% \mathrm{w} / \mathrm{v}$ yeast at $60 \%$ relative humidity.

Survival experiments. All Drosophila survival experiments were performed with mated female flies at $23^{\circ} \mathrm{C}$. Throughout the lifespan assessment, flies were kept in vials in groups of 10 and transferred to a new food vial every second or third day. The number of surviving flies was counted after each transfer. Average lifespan was calculated using the DABEST estimation statistics package ${ }^{91}$. The data were plotted to compare the average survival of each tested genotype against the average survival of the $w[1118]$ control stock that was assayed in parallel.

The MOT. The MOT apparatus was designed to allow the monitoring of Drosophila behavior throughout olfactory conditioning in a controlled environment ${ }^{16}$. Flies were assayed in conditioning chambers, whereby the arena of each chamber was $50 \mathrm{~mm}$ long, $5 \mathrm{~mm}$ wide, and $1.3 \mathrm{~mm}$ high (Supplementary Fig. 2a). The floor and ceiling of each chamber was composed of a glass slide printed with transparent indium tin oxide electrodes (Walthy, China). Each side of the electrode board was sealed by a gasketed lid that formed a seal around the gap between the electrode board and the chamber wall. Facilitated by carrier air, the odors entered the chamber via two entry pipes and left the chamber through two vents that were located in the middle of the chamber. Up to four MOT chambers were stacked onto a rack which was connected to the odor and electric shock supply (Supplementary Fig. 2b). Chambers were illuminated from the back by two grids of infrared LEDs. Behavior inside the chambers was recorded with an AVT F-080 Guppy camera (Allied Vision) that was connected to a video acquisition board (PCI-1409, National Instruments). Electric shock during odor presentation is delivered when the animals walk on the electrode contacts. Olfactory preference was measured by tracking the movement of individual flies and scored automatically by using a custom tracking and control program (CRITTA) ${ }^{92}$.

Odor delivery and odor concentrations. Odor delivery in the MOT was done as previously described ${ }^{16}$, with some protocol modifications. The rack with stacked conditioning chambers was connected to an olfactometer that was used to deliver precisely timed odor stimuli (Supplementary Fig. $2 \mathrm{~b}$ ). The conditioning odors methylcyclohexanol (MCH) and 3-octanol (OCT) were carried by dry, compressed air and routed through mass flow controllers (MFC; Sensirion AG). Carrier air flow was controlled with two $2 \mathrm{~L} / \mathrm{min}$ capacity MFCs and pushed through a humidifying gas washing bottle containing distilled water (Schott Duran) at $0.6 \mathrm{~L} / \mathrm{min}$. Odor streams were controlled with $500 \mathrm{~mL} / \mathrm{min}$ MFCs and pushed through glass vials containing pure liquid odorants (either $\mathrm{MCH}$ or OCT, respectively). Prior to conditioning, the odor concentrations were adjusted to ensure that flies did not display a strong preference for one of the odors over the other prior to training. Odor administration was carried out with the following MFC settings: OCT left side $25-35 \mathrm{~mL} / \mathrm{min}$; OCT right side $30-40 \mathrm{~mL} / \mathrm{min}$; MCH left side $50-60 \mathrm{~mL} / \mathrm{min}$; 
$\mathrm{MCH}$ right side $50-60 \mathrm{~mL} / \mathrm{min}$. Odor presentation at the behavioral chamber arms was switched with computer-controlled solenoid valves (The Lee Company, USA). The MFCs were regulated via CRITTA (LabView software). At ad hoc intervals between experiments, odor concentrations were measured with a photoionization detector (PID, RAE systems; PGM-7340). The experiments were performed with a relative concentration of 14-16 parts per million (ppm) for $\mathrm{MCH}$ and 6-8 ppm for OCT in the chambers. A relative humidity of $70-75 \%$ was maintained via regulation of the air flow; this was monitored (ad hoc, between experiments) by using a custom humidity sensor with a custom LabVIEW code (National Instruments, USA).

Olfactory conditioning and data visualization. Classical olfactory conditioning has been described previously $y^{5,6,16}$. Before each experiment, flies were briefly anesthetized on ice and six flies were loaded into each conditioning chamber (Supplementary Fig. 2A-B). Each conditioning experiment began with an acclimatization (baseline test) phase where Drosophila were exposed to both odors in the absence of a shock stimulus. Subsequently, in the first stage of training the chambers were flushed with carrier air and flies were exposed to either $\mathrm{MCH}$ or OCT in the presence of a shock stimulus (12 shocks at $60 \mathrm{~V}$ during a $60-\mathrm{s}$ time interval). During the second stage of training the shock-paired odor was removed and the flies were exposed to the other odor in the absence of shock. After removal of the odor and air-puff agitation, flies were tested for shock-odor avoidance. The flies were given a choice between the two odors and average shock-odor avoidance was quantified for the last $30 \mathrm{~s}$ of the 2 min-long testing phase. The main stages of the conditioning protocol are summarized in Fig. $2 \mathrm{~b}$. The full conditioning protocol is presented in Supplementary Fig. 2d. The shock-odor avoidance of flies for each conditioning trial was expressed as a performance index $(\mathrm{PI})^{6}$; however, instead of a single endpoint, counting was performed on individual video frames over the final $30 \mathrm{~s}$ of the testing period. Each trial produced a half PI against the respective conditioned odor (either MCH or OCT) and two half PI's from consecutive experiments (with different conditioning odors) were combined to a full PI (full PI $=$ half PI OCT + half PI MCH). For data visualization, the distribution of full PI's was plotted with a $95 \% \mathrm{CI}$ error presenting a $\triangle \mathrm{PI}$ between control and test genotypes by using the DABEST estimation-statistics package ${ }^{91}$.

Behavioral data analysis. Analyses of STM and survival experiments were performed with estimation statistics ${ }^{93-95}$. The rationale for the estimation framework was described previously ${ }^{91,96}$. For data analysis and visualization, the individual values of full PIs were plotted with standard-deviation lines; to describe the differences between control and test genotypes, the distributions of $\Delta$ PI with $95 \%$ confidence intervals were plotted with the DABEST estimation package (www. estimationstats.com). Using this approach, we avoid null-hypothesis significance testing ${ }^{97,98}$ in favor of estimation plots, which show the relevant effect sizes and comprehensive distributional information of both observed and inferred values, focusing on the intervention effect size. For legacy purposes only, the two-group comparison permutation $P$ values are listed in the respective legends for each figure. The sample size of at least 72 flies per group for behavioral experiments was based on precision planning to accommodate an average target margin of error of 0.33 standardized effect-size units ${ }^{94}$. This is equivalent to $80 \%$ power to detect a 0.5 SD effect size (https://www.esci.thenewstatistics.com/esci-precision.html\#tab-1).

Drosophila immunostaining. We performed immunostaining as previously described $^{99}$, by fixing dissected tissues in PBS containing $4 \%$ formaldehyde and incubating with the following primary antibodies: mouse $\alpha$-HA (1:1000, Santa Cruz), and guinea pig $\alpha$-Mettl3 (1:2000, gift of Cintia Hongay, Clarkson University). Alexa Fluor-488, and -568 secondary antibodies were from Molecular Probes and used at 1:1000. Tissues were mounted in Vectashield mounting buffer with DAPI (Vector Laboratories). Images were captured with a Leica SP5 confocal microscope; endogenous GFP signals were monitored.

\section{$m^{6} \mathbf{A}$ individual-nucleotide-resolution cross-linking and immunoprecipitation} (miCLIP). miCLIP libraries were prepared by subjecting RNA samples to the established protocol ${ }^{66}$ with the minor changes described below. Briefly, total RNA was collected from <1-week-old w1118 (wild type) and Mettl3[null] (mutant) female heads using TRIzol RNA extraction. Poly(A) + RNA was enriched using two rounds of selection. RNAs were fragmented, incubated with $a-m^{6} \mathrm{~A}(202003$ Synaptic Systems) and crosslinked twice in a Stratalinker 2400 (Stratagene) using $150 \mathrm{~mJ} / \mathrm{cm}^{2}$. Crosslinked RNAs were immunoprecipitated using Protein A/G magnetic beads (Thermo) and washed under high salt conditions to reduce nonspecific binding. Samples were radiolabeled with T4 PNK (NEB), ligated to a $3^{\prime}$ adaptor using T4 RNA Ligase I (NEB), and purified using SDS-polyacrylamide gel electrophoresis (SDS-PAGE) and nitrocellulose membrane transfer. RNA fragments containing crosslinked antibody peptides were recovered from the membrane using proteinase $\mathrm{K}$ (Invitrogen) digestion.

Recovered fragments were subjected to library preparation. First-strand cDNA synthesis was performed using SuperScript III (Life Technologies) and iCLIPbarcoded primers, which contain complementarity to the $3^{\prime}$ adaptor on the RNA. cDNAs were purified using denaturing PAGE purification, circularized using CircLigase II (EpiCentre), annealed to the iCLIP Cut Oligo, and digested using
BamHI (Thermo). To generate libraries for sequencing, the resulting linear cDNAs were amplified using Accuprime SuperMix I (Invitrogen) and P5 and P3 Solexa primers, and purified using Agencourt AMPure XP beads (Beckman Coulter).

For input libraries, poly(A) + RNAs were fragmented and directly subjected to radiolabelling and $3^{\prime}$ adaptor ligation. All subsequent steps are as listed above. Libraries were paired-end sequenced on an Illumina HiSeq2500 instrument at the New York Genome Center (NYGC).

miCLIP bioinformatic analyses. Read processing, mutation calling, and annotation of CIMs was performed as described ${ }^{66}$. Briefly, to prepare libraries for mapping, adapters and low-quality reads were trimmed using flexbar v2.5. Next, the FASTQ files were de-multiplexed using the pyBarcodeFilter.py script from the pyCRAC suite. Random barcodes were removed from sequencing reads and appended to sequence IDs using an awk script and PCR duplicates were removed using the pyCRAC pyDuplicateRemover.py script. Paired end reads were merged and mapped to the Drosophila reference genome sequence (BDGP Release 6/dm6) using Novoalign (Novocraft) with parameters $-\mathrm{t} 85$ and -116 .

Mutations were called using the CIMS software package ${ }^{100}$. To identify putative $\mathrm{m}^{6} \mathrm{~A}$ sites, C-to-T transitions with preceding A nucleotides were extracted and filtered such that the number of mutations that support the mismatch $(m)>1$ and $0.01<m / k<0.5$, where $k$ is the number of unique tags that span the mismatch position.

Peaks were called by adapting the model-based analysis for ChIP-Seq (MACS) algorithm ${ }^{101}$. Mettl3-dependent peaks for head libraries were determined using miCLIP versus input, comparing wild type and Mettl3 libraries and the MACS2 differential binding events program (bdgdiff) with parameters $-\mathrm{g} 20$ and -1120 . Lastly, peaks were split using PeakSplitter (version 1.0, http://www.ebi.ac.uk/ research/bertone/software).

To generate nucleotide content plots, filtered C-to-T transitions with preceding A nucleotide (as mentioned above) that mapped within the top 100 or 1000 Mettl3dependent peaks were chosen to describe the nucleotide content surrounding CIMs. Sequences were obtained using the Drosophila reference genome sequence (dm6) and fed to WebLogo version 2.8.2 with the frequency setting ${ }^{102}$.

Custom scripts were used to generate metagene plots. Briefly, to prepare mapped data, each miCLIP bam file was converted to bedGraph format with span of 1 nucleotide. To prepare features, for each gene, the longest transcript model was selected and divided into $5^{\prime}$ UTR, CDS and $3^{\prime}$ UTR segments according to Ensembl transcript models for BDGP6.94. Next, miCLIP read depth mapping to transcripts were selected and scaled such that each $5^{\prime}$ UTR, CDS and $3^{\prime}$ UTR were 200, 1000 and 300 nts. To normalize, the score at each scaled nucleotide was divided by the total score across all 1500 nucleotides. Finally, to yield metagene score across each feature (UTRs and CDS), genes of interest were selected and means were calculated for each nucleotide position. Smoothing functions from the ggplot2 package ${ }^{103}$ were used to visualize metagene analysis.

Pie charts were obtained by mapping peaks to Ensembl transcript models for BDGP 6.94. Since transcript features occasionally overlap, the following order was used to bin peaks into different categories: other (not mapping transcript models) introns, start codons, 5' UTRs, 3' UTRs, and CDS. Finally, the ggplot2 function geom_bar was used to plot the accounted annotations into a pie chart.

Input normalized miCLIP tracks along with described peaks were used to generate heatmaps using deepTools2 functions computeMatrix and plotHeatmap ${ }^{104}$

RNA-seq analysis. Flies of the specified genotypes $(w ; ; \operatorname{Mettl3} 3$ null $] /+, w ; ;$ Mettl3 [null]/Mettl3[cat], w;;Df(3 R)BSC461/ +, w;;Ythdf[NP3]/Df(3 R)BSC461, were aged to 1 or 3 weeks at $25^{\circ} \mathrm{C}$. Female heads were dissected and collected for total RNA extraction using TRIzol reagent. Sequencing libraries were prepared using the TruSeq Stranded Total RNA Sequencing Kit (Illumina) following the manufacturer's protocol. Sequencing was performed on a HiSeq 2500 System in paired end read mode, with 100 bases per read at the Integrated Genomics Operation (IGO) at Memorial Sloan Kettering Cancer Center.

RNA sequencing libraries were mapped to the Drosophila reference genome sequence (BDGP Release 6/dm6) using HISAT2 ${ }^{105}$ under the default settings. Gene counts were obtained by assigning and counting reads to the Ensembl transcript models for BDGP6.94 using Rsubread ${ }^{106}$. Differential gene expression analysis was performed with comparisons as listed in Supplementary Dataset 5 using the $\mathrm{R}$ package DESeq2 ${ }^{107}$ and applying a strict adjusted $p$-value cutoff of 0.05 .

CRISPR/Cas9 deletion of Mett/3 in S2-S cells. We used CRISPR/Cas9-mediated mutagenesis as described ${ }^{67}$ to generate Mettl3-KO S2 cell lines. Guide RNA sequences are listed in Supplementary Dataset 6. We analyzed 11 candidate clonal lines obtained from subcloning of two initial low-complexity mixed cell populations, and kept the deletions \#3-5 and \#4-3 as described in Supplementary Fig. 11.

$\mathbf{m}^{\mathbf{6}} \mathbf{A}$-RIP-PCR and RIP-rtPCR. We adapted a protocol from our recent study ${ }^{55}$. Plasmids of $5 \mu \mathrm{g}$ were transfected into $6 \times 10$ [6] S2 cells using Effectene (Qiagen) and incubated for 3 days. Cells were washed with PBS and lysed with IP lysis buffer (30 mM HEPES, pH 7.5, $150 \mathrm{mM}$ KOAc, $2 \mathrm{mM} \mathrm{Mg}(\mathrm{OAc})_{2}, 5 \mathrm{mM}$ DTT, $0.1 \%$ NP40) supplied with Complete, EDTA-free Protease Inhibitor and $40 \mathrm{U} \mathrm{mL}^{-1}$ SUPERase.In RNase Inhibitor (Ambion) on ice for $30 \mathrm{~min}$, followed by $2 \times 10 \mathrm{~min}$ 
centrifugation at $20,000 \times g$ at $4{ }^{\circ} \mathrm{C} .10 \%$ of the cleared cell lysate were kept as input and the rest was incubated with $15 \mu \mathrm{L}$ Dynabeads ${ }^{\mathrm{mm}}$ Protein G (Thermo Fisher,10004D) (with HA or GFP antibody) for $4 \mathrm{~h}$ at $4{ }^{\circ} \mathrm{C}$. RNase I (Invitrogen, AM2294) was added to the sample for RNase treatment at $0.2 \mathrm{U}$ final concentration. The beads were washed three times using IP lysis buffer and then resuspended in $100 \mathrm{~mL}$ lysis buffer. To elute RNA, the beads were mixed with $900 \mathrm{~mL}$ of Trizol, vortexed for $1 \mathrm{~min}$ and incubated at RT for 50 with rotation. RNA extracted and treated were Turbo DNase (Ambion) for $30 \mathrm{~min}$ before cDNA synthesis using SuperScript III (Life technology) with random hexamers. PCRs were done using fusion high-fidelity polymerase (ThermoFisher Scientific).

For $\mathrm{m}^{6} \mathrm{~A}-\mathrm{RIP}-\mathrm{qPCR}$, the mRNAs were immunoprecipitated using $a-\mathrm{m}^{6} \mathrm{~A}$ according to the procedure shown above. The IP-mRNAs were then reverse transcribed and amplified following the same protocol. The enrichment of $\mathrm{m}^{6} \mathrm{~A}$ was quantified using $\mathrm{qPCR}$ as reported. The sequences of $\mathrm{qPCR}$ primers are listed in Supplementary Dataset 6.

RNA degradation assay. S2 cells were seeded as $3 \times 10^{6}$ cells per well. Actinomycin-D (Gibco, 11-805-017) was added to a final concentration of $5 \mu \mathrm{M}$, and cells were collected before or $5 \mathrm{~h}$ after adding actinomycin-D. Then the cells were processed as described in 'RT-qPCR', except that the data were normalized to the $t=0$ time point.

SUnSET assay and Western blotting. For each assay, we incubated $3 \times 10[6]$ cells in $1 \mathrm{~mL}$ Schneider's medium including $10 \% \mathrm{FBS}$ for $5 \mathrm{~min}$ at $25^{\circ} \mathrm{C}$ with or without $5 \mu \mathrm{g} / \mathrm{mL}$ puromycin (Gibco ${ }^{\mathrm{m}}$ Sterile Puromycin Dihydrochloride). Cells were then washed twice with cold PBS, and lysed with $100 \mu \mathrm{L}$ lysis buffer $(10 \mathrm{mM}$ Tris- $\mathrm{HCl}$, pH 7.5, $300 \mathrm{mM} \mathrm{NaCl}, 1 \mathrm{mM}$ EDTA, 1\% Triton X-100, Protease Inhibitor Cocktail Roche). The cell pellet was resuspended by pipetting and incubated on ice for $30 \mathrm{~min}$, then centrifuged at $16,000 \times \mathrm{g}$ for $10 \mathrm{~min}$ at $4^{\circ} \mathrm{C}$. Protein concentration was measured using Bio-Rad Protein Assay Dye (500-0006) and $2.5 \mu \mathrm{g}$ proteins were separated on SDS-PAGE and transferred to Immobilon-P membranes. Membranes were blocked for $1 \mathrm{~h}$ in TBS containing 5\% nonfat milk and $0.1 \%$ Tween-20, followed by incubation with mouse $\alpha$-puromycin $(1: 1000)$ overnight at $4{ }^{\circ} \mathrm{C}$. Appropriate secondary antibodies conjugated to HRP (Jackson) were used at 1:5000 for $1 \mathrm{~h}$ at room temperature, then visualized using chemiluminescence detection (Amersham ECL Prime Western Blotting Detection Reagent). Mouse $\alpha$ puromycin $(2 \mathrm{~A} 4,1: 1000)$ and mouse $\alpha-\beta$-tubulin (E7, 1:1000) were from DSHB; guinea pig $\alpha$-Mettl3 (1:5000) was a gift from Cintia Hongay.

Luciferase sensory assays. To generate the pAc5.1-aqz-wt-luc reporter, we cloned the $a q z 5^{\prime}$ UTR (chr3R:8,818,731-8,820,682) into the $5^{\prime}$ UTR pAc5.1-luc vector encoding Firefly Luciferase (KpnI/EcoRI). We then used site-directed mutagenesis (primers listed in Supplementary Dataset 6) to generate pAc5.1-aqzM-luc (8820387A, 8820401A into T).

To assay luciferase reporter activity and mRNA levels, $10^{6} \mathrm{~S} 2$ cells were seeded per well of 12-well plate and transfected with $150 \mathrm{ng}$ pAc5.1-Renilla, and $150 \mathrm{ng}$ pAc5.1-aqz-wt-luc, or pAc5.1-aqz-M-luc constructs. Luciferase activities were measured 3 days after transfection using Dual Glo luciferase assay system (Promega) and Cytation5 (BioTek) from $100 \mu \mathrm{L}$ cell from each well of the 12-well plate. We calculated the ratio between Firefly and Renilla luciferase activities. The remaining cells in the 12-well plate were processed to extract total RNA (DNase I digested) by TRIzol ${ }^{\oplus}$ reagent (Invitrogen), followed by RT-qPCR quantification. The level of $F-l u c$ mRNA was normalized by that of $R$-luc mRNA.

Reporting summary. Further information on research design is available in the Nature Research Reporting Summary linked to this article.

\section{Data availability}

All transgenic strains and plasmids generated for this study are available upon request. All of the raw miCLIP and RNA-seq data generated in this study were deposited in NCBI-GEO under GSE147230. Source data are provided with this paper. All of the raw and summarized STM, locomotion, and survival data are available from the zenodo repository under the following DOI: 10.5281 /zenodo.4446416. Source data are provided with this paper.

Received: 10 March 2020; Accepted: 28 January 2021;

Published online: 05 March 2021

\section{References}

1. Dudai, Y., Jan, Y. N., Byers, D., Quinn, W. G. \& Benzer, S. dunce, a mutant of Drosophila deficient in learning. Proc. Natl Acad. Sci. USA 73, 1684-1688 (1976).

2. Vosshall, L. B. Into the mind of a fly. Nature 450, 193-197 (2007).
3. Kahsai, L. \& Zars, T. Learning and memory in Drosophila: behavior, genetics, and neural systems. Int. Rev. Neurobiol. 99, 139-167 (2011).

4. Keene, A. C. \& Waddell, S. Drosophila olfactory memory: single genes to complex neural circuits. Nat. Rev. Neurosci. 8, 341-354 (2007).

5. Tumkaya, T., Ott, S. \& Claridge-Chang, A. A systematic review of Drosophila short-term-memory genetics: meta-analysis reveals robust reproducibility. Neurosci. Biobehav. Rev. 95, 361-382 (2018).

6. Tully, T. \& Quinn, W. G. Classical conditioning and retention in normal and mutant Drosophila melanogaster. J. Comp. Physiol. A 157, 263-277 (1985).

7. Busto, G. U., Guven-Ozkan, T., Fulga, T. A., Van Vactor, D. \& Davis, R. L. microRNAs that promote or inhibit memory formation in Drosophila melanogaster. Genetics 200, 569-580 (2015).

8. Guven-Ozkan, T. \& Davis, R. L. Functional neuroanatomy of Drosophila olfactory memory formation. Learn Mem. 21, 519-526 (2014).

9. de Belle, J. S. \& Heisenberg, M. Associative odor learning in Drosophila abolished by chemical ablation of mushroom bodies. Science 263, 692-695 (1994).

10. Tanaka, N. K., Awasaki, T., Shimada, T. \& Ito, K. Integration of chemosensory pathways in the Drosophila second-order olfactory centers. Curr. Biol. 14, 449-457 (2004).

11. Clyne, P. J. et al. A novel family of divergent seven-transmembrane proteins: candidate odorant receptors in Drosophila. Neuron 22, 327-338 (1999).

12. Vosshall, L. B., Amrein, H., Morozov, P. S., Rzhetsky, A. \& Axel, R. A spatial map of olfactory receptor expression in the Drosophila antenna. Cell 96, 725-736 (1999).

13. Gao, Q., Yuan, B. \& Chess, A. Convergent projections of Drosophila olfactory neurons to specific glomeruli in the antennal lobe. Nat. Neurosci. 3, 780-785 (2000).

14. Fishilevich, E. \& Vosshall, L. B. Genetic and functional subdivision of the Drosophila antennal lobe. Curr. Biol. 15, 1548-1553 (2005).

15. Kitamura, T. et al. Engrams and circuits crucial for systems consolidation of a memory. Science 356, 73-78 (2017).

16. Claridge-Chang, A. et al. Writing memories with light-addressable reinforcement circuitry. Cell 139, 405-415 (2009).

17. Yamagata, N. et al. Distinct dopamine neurons mediate reward signals for short- and long-term memories. Proc. Natl Acad. Sci. USA 112, 578-583 (2015).

18. Liu, C. et al. A subset of dopamine neurons signals reward for odour memory in Drosophila. Nature 488, 512-516 (2012).

19. Zaccara, S., Ries, R. J. \& Jaffrey, S. R. Reading, writing and erasing mRNA methylation. Nat. Rev. Mol. Cell Biol. 20, 608-624 (2019).

20. Perry, R. P. \& Kelly, D. E. Existence of methylated messenger RNA in mouse L cells. Cell 1, 37-42 (1974).

21. Desrosiers, R., Friderici, K. \& Rottman, F. Identification of methylated nucleosides in messenger RNA from Novikoff hepatoma cells. Proc. Natl Acad. Sci. USA 71, 3971-3975 (1974)

22. Patil, D. P., Pickering, B. F. \& Jaffrey, S. R. Reading m(6)A in the transcriptome: m(6)A-binding proteins. Trends Cell Biol. 28, 113-127 (2018).

23. Luo, S. \& Tong, L. Molecular basis for the recognition of methylated adenines in RNA by the eukaryotic YTH domain. Proc. Natl Acad. Sci. USA 111, 13834-13839 (2014)

24. Theler, D., Dominguez, C., Blatter, M., Boudet, J. \& Allain, F. H. Solution structure of the YTH domain in complex with N6-methyladenosine RNA: a reader of methylated RNA. Nucleic Acids Res. 42, 13911-13919 (2014).

25. Xu, C. et al. Structural basis for the discriminative recognition of N6methyladenosine RNA by the human YT521-B homology domain family of proteins. J. Biol. Chem. 290, 24902-24913 (2015).

26. Xu, C. et al. Structural basis for selective binding of m6A RNA by the YTHDC1 YTH domain. Nat. Chem. Biol. 10, 927-929 (2014).

27. Xiao, W. et al. Nuclear m(6)A reader YTHDC1 regulates mRNA splicing. Mol. Cell 61, 507-519 (2016).

28. Roundtree, I. A. et al. YTHDC1 mediates nuclear export of N(6)methyladenosine methylated mRNAs. eLife 6, e31311 (2017).

29. Wang, X. et al. N6-methyladenosine-dependent regulation of messenger RNA stability. Nature 505, 117-120 (2014).

30. $\mathrm{Du}, \mathrm{H}$. et al. YTHDF2 destabilizes $\mathrm{m}(6) \mathrm{A}$-containing RNA through direct recruitment of the CCR4-NOT deadenylase complex. Nat. Commun. 7, 12626 (2016).

31. Ries, R. J. et al. m(6)A enhances the phase separation potential of mRNA. Nature 571, 424-428 (2019).

32. Zaccara, S. \& Jaffrey, S. R. A unified model for the function of YTHDF proteins in regulating m(6)A-modified mRNA. Cell 181, 1582-1595 (2020). e1518.

33. Wang, X. et al. N(6)-methyladenosine modulates messenger RNA translation efficiency. Cell 161, 1388-1399 (2015).

34. Shi, H. et al. m(6)A facilitates hippocampus-dependent learning and memory through YTHDF1. Nature 563, 249-253 (2018).

35. Shi, H. et al. YTHDF3 facilitates translation and decay of $\mathrm{N}(6)$ methyladenosine-modified RNA. Cell Res. 27, 315-328 (2017). 
36. Zhou, J. et al. Dynamic m(6)A mRNA methylation directs translational control of heat shock response. Nature 526, 591-594 (2015).

37. Zhang, Z. et al. Genetic analyses support the contribution of mRNA N(6)methyladenosine $(\mathrm{m}(6) \mathrm{A})$ modification to human disease heritability. Nat. Genet. 52, 939-949 (2020)

38. Mao, Y. et al. $\mathrm{m}(6) \mathrm{A}$ in mRNA coding regions promotes translation via the RNA helicase-containing YTHDC2. Nat. Commun. 10, 5332 (2019).

39. Livneh, I., Moshitch-Moshkovitz, S., Amariglio, N., Rechavi, G. \& Dominissini, D. The m(6)A epitranscriptome: transcriptome plasticity in brain development and function. Nat. Rev. Neurosci. 21, 36-51 (2020).

40. Yoon, K. J. et al. Temporal control of mammalian cortical neurogenesis by $\mathrm{m}$ (6)A methylation. Cell 171, 877-889 (2017). e817.

41. Wang, C. X. et al. METTL3-mediated m6A modification is required for cerebellar development. PLoS Biol. 16, e2004880 (2018)

42. Ma, C. et al. RNA m(6)A methylation participates in regulation of postnatal development of the mouse cerebellum. Genome Biol. 19, 68 (2018).

43. Li, M. et al. Ythdf2-mediated m(6)A mRNA clearance modulates neural development in mice. Genome Biol. 19, 69 (2018).

44. Weng, Y. L. et al. Epitranscriptomic m(6)A regulation of axon regeneration in the adult mammalian nervous system. Neuron 97, 313-325 (2018). e316.

45. Wang, Y. et al. N(6)-methyladenosine RNA modification regulates embryonic neural stem cell self-renewal through histone modifications. Nat. Neurosci. 21, 195-206 (2018).

46. Zhang, Z. et al. METTL3-mediated N(6)-methyladenosine mRNA modification enhances long-term memory consolidation. Cell Res. 28, 1050-1061 (2018)

47. Hess, M. E. et al. The fat mass and obesity associated gene (Fto) regulates activity of the dopaminergic midbrain circuitry. Nat. Neurosci. 16, 1042-1048 (2013).

48. Widagdo, J. et al. Experience-dependent accumulation of N6-methyladenosine in the prefrontal cortex is associated with memory processes in mice. $J$. Neurosci. 36, 6771-6777 (2016).

49. Koranda, J. L. et al. Mettl14 is essential for epitranscriptomic regulation of striatal function and learning. Neuron 99, 283-292 (2018). e285.

50. Walters, B. J. et al. The role of the RNA demethylase FTO (fat mass and obesity-associated) and mRNA methylation in hippocampal memory formation. Neuropsychopharmacology 42, 1502-1510 (2017).

51. Dezi, V., Ivanov, C., Haussmann, I. U. \& Soller, M. Nucleotide modifications in messenger RNA and their role in development and disease. Biochem. Soc. Trans. 44, 1385-1393 (2016).

52. Hongay, C. F. \& Orr-Weaver, T. L. Drosophila Inducer of MEiosis 4 (IME4) is required for Notch signaling during oogenesis. Proc. Natl Acad. Sci. USA 108, 14855-14860 (2011)

53. Haussmann, I. U. et al. m6A potentiates Sxl alternative pre-mRNA splicing for robust Drosophila sex determination. Nature 540, 301-304 (2016).

54. Lence, T. et al. m6A modulates neuronal functions and sex determination in Drosophila. Nature 540, 242-247 (2016).

55. Kan, L. et al. The m(6)A pathway facilitates sex determination in Drosophila. Nat. Commun. 8, 15737 (2017).

56. Arguello, A. E., Leach, R. W. \& Kleiner, R. E. In vitro selection with a sitespecifically modified RNA library reveals the binding preferences of N(6)methyladenosine reader proteins. Biochemistry 58, 3386-3395 (2019).

57. Arguello, A. E., DeLiberto, A. N. \& Kleiner, R. E. RNA chemical proteomics reveals the $\mathrm{N}(6)$-methyladenosine $(\mathrm{m}(6) \mathrm{A})$-regulated protein-RNA interactome. J. Am. Chem. Soc. 139, 17249-17252 (2017).

58. Edupuganti, R. R. et al. N(6)-methyladenosine (m(6)A) recruits and repels proteins to regulate mRNA homeostasis. Nat. Struct. Mol. Biol. 24, 870-878 (2017).

59. $\mathrm{Wu}, \mathrm{R}$. et al. A novel $\mathrm{m}(6) \mathrm{A}$ reader Prrc2a controls oligodendroglial specification and myelination. Cell Res. 29, 23-41 (2019).

60. Huang, $H$. et al. Recognition of RNA N(6)-methyladenosine by IGF2BP proteins enhances mRNA stability and translation. Nat. Cell Biol. 20, 285-295 (2018).

61. Linder, B. et al. Single-nucleotide-resolution mapping of m6A and m6Am throughout the transcriptome. Nat. Methods 12, 767-772 (2015).

62. Pendleton, K. E. et al. The U6 snRNA m(6)A methyltransferase METTL16 regulates SAM synthetase intron retention. Cell 169, 824-835 (2017). e814.

63. Salmon-Divon, M., Dvinge, H., Tammoja, K. \& Bertone, P. PeakAnalyzer: genome-wide annotation of chromatin binding and modification loci. BMC Bioinforma. 11, 415 (2010).

64. Zhao, B. S., Roundtree, I. A. \& He, C. Post-transcriptional gene regulation by mRNA modifications. Nat. Rev. Mol. Cell Biol. 18, 31-42 (2017).

65. Zhang, C. et al. m(6)A modulates haematopoietic stem and progenitor cell specification. Nature 549, 273-276 (2017).

66. Grozhik, A. V., Linder, B., Olarerin-George, A. O. \& Jaffrey, S. R. Mapping m (6)A at individual-nucleotide resolution using crosslinking and immunoprecipitation (miCLIP). Methods Mol. Biol. 1562, 55-78 (2017).

67. Reimao-Pinto, M. M. et al. Molecular basis for cytoplasmic RNA surveillance by uridylation-triggered decay in Drosophila. EMBO J. 35, 2417-2434 (2016).
68. Cheng, Y. et al. m(6)A RNA methylation maintains hematopoietic stem cell identity and symmetric commitment. Cell Rep. 28, 1703-1716 (2019). e1706.

69. Burow, D. A. et al. Dynamic regulation of mRNA decay during neural development. Neural Dev. 10, 11 (2015).

70. Zhang, H. et al. Genome-wide maps of ribosomal occupancy provide insights into adaptive evolution and regulatory roles of uORFs during Drosophila development. PLoS Biol. 16, e2003903 (2018).

71. Schmidt, E. K., Clavarino, G., Ceppi, M. \& Pierre, P. SUnSET, a nonradioactive method to monitor protein synthesis. Nat. Methods 6, 275-277 (2009).

72. Mendoza-Ortiz, M. A., Murillo-Maldonado, J. M. \& Riesgo-Escovar, J. R. aaquetzalli is required for epithelial cell polarity and neural tissue formation in Drosophila. PeerJ 6, e5042 (2018).

73. Brennecke, J., Stark, A., Russell, R. B. \& Cohen, S. M. Principles of microRNAtarget recognition. PLoS Biol. 3, e85 (2005).

74. Bollepalli, M. K., Kuipers, M. E., Liu, C. H., Asteriti, S. \& Hardie, R. C. Phototransduction in Drosophila is compromised by Gal4 expression but not by InsP3 receptor knockdown or mutation. eNeuro 4, e0143-17 (2017).

75. Kramer, J. M. \& Staveley, B. E. GAL4 causes developmental defects and apoptosis when expressed in the developing eye of Drosophila melanogaster. Genet Mol. Res. 2, 43-47 (2003).

76. Heisenberg, M., Borst, A., Wagner, S. \& Byers, D. Drosophila mushroom body mutants are deficient in olfactory learning. J. Neurogenet. 2, 1-30 (1985).

77. Knuckles, P. et al. Zc3h13/Flacc is required for adenosine methylation by bridging the mRNA-binding factor Rbm15/Spenito to the m(6)A machinery component Wtap/Fl(2)d. Genes Dev. 32, 415-429 (2018).

78. Guo, J., Tang, H. W., Li, J., Perrimon, N. \& Yan, D. Xio is a component of the Drosophila sex determination pathway and RNA N(6)-methyladenosine methyltransferase complex. Proc. Natl Acad. Sci. USA 115, 3674-3679 (2018)

79. Sommer, S., Lavi, U. \& Darnell, J. E. Jr. The absolute frequency of labeled N-6methyladenosine in HeLa cell messenger RNA decreases with label time. J. Mol. Biol. 124, 487-499 (1978).

80. Wang, Y. et al. N6-methyladenosine modification destabilizes developmental regulators in embryonic stem cells. Nat. Cell Biol. 16, 191-198 (2014).

81. Ke, S. et al. m(6)A mRNA modifications are deposited in nascent pre-mRNA and are not required for splicing but do specify cytoplasmic turnover. Genes Dev. 31, 990-1006 (2017).

82. Herzog, V. A. et al. Thiol-linked alkylation of RNA to assess expression dynamics. Nat. Methods 14, 1198-1204 (2017).

83. Meyer, K. D. et al. 5' UTR m(6)A promotes cap-independent translation. Cell 163, 999-1010 (2015)

84. Choe, J. et al. mRNA circularization by METTL3-eIF3h enhances translation and promotes oncogenesis. Nature 561, 556-560 (2018)

85. Quinn, W. G. \& Dudai, Y. Memory phases in Drosophila. Nature 262, 576-577 (1976)

86. Isabel, G., Pascual, A. \& Preat, T. Exclusive consolidated memory phases in Drosophila. Science 304, 1024-1027 (2004).

87. Giese, K. P. \& Mizuno, K. The roles of protein kinases in learning and memory. Learn Mem. 20, 540-552 (2013)

88. Button, K. S. et al. Power failure: why small sample size undermines the reliability of neuroscience. Nat. Rev. Neurosci. 14, 365-376 (2013).

89. Tamura, T. et al. Aging specifically impairs amnesiac-dependent memory in Drosophila. Neuron 40, 1003-1011 (2003)

90. Su, D. et al. Quantitative analysis of ribonucleoside modifications in tRNA by HPLC-coupled mass spectrometry. Nat. Protoc. 9, 828-841 (2014).

91. Ho, J., Tumkaya, T., Aryal, S., Choi, H. \& Claridge-Chang, A. Moving beyond P values: data analysis with estimation graphics. Nat. methods 16, 565-566 (2019).

92. Krishnan, S. et al. The right dorsal habenula limits attraction to an odor in zebrafish. Curr. Biol. 24, 1167-1175 (2014).

93. Altman, D., Machin, D., Bryant, T. \& Gardner, S. Statistics with Confidence: Confidence Interval and Statistical Guidelines (BMJ Books, 2000).

94. Cumming, G. Understanding the New Statistics Effect Sizes, Confidence Intervals, and Meta-analysis (Routledge, 2012)

95. Cumming, G. \& Calin-Jageman, R. Introduction to the New Statistics: Estimation, Open Science, and Beyond (Routledge, 2016).

96. Claridge-Chang, A. \& Assam, P. N. Estimation statistics should replace significance testing. Nat. Methods 13, 108-109 (2016).

97. Wasserstein, R. L., Schirm, A. L. \& Lazar, N. A. Moving to a world beyond "p < 0.05.". Am. Stat. 73, 1-19 (2019).

98. Halsey, L. G., Curran-Everett, D., Vowler, S. L. \& Drummond, G. B. The fickle $P$ value generates irreproducible results. Nat. Methods 12, 179-185 (2015).

99. Lai, E. C. \& Rubin, G. M. neuralized functions cell-autonomously to regulate a subset of Notch-dependent processes during adult Drosophila development. Dev. Biol. 231, 217-233 (2001).

100. Zhang, C. \& Darnell, R. B. Mapping in vivo protein-RNA interactions at single-nucleotide resolution from HITS-CLIP data. Nat. Biotechnol. 29, 607-614 (2011).

101. Zhang, Y. et al. Model-based analysis of ChIP-Seq (MACS). Genome Biol. 9, R137 (2008). 
102. Crooks, G. E., Hon, G., Chandonia, J. M. \& Brenner, S. E. WebLogo: a sequence logo generator. Genome Res. 14, 1188-1190 (2004).

103. Wickham, H. ggplot2: Elegant Graphics for Data Analysis (Springer-Verlag, 2016).

104. Ramirez, F. et al. deepTools2: a next generation web server for deepsequencing data analysis. Nucleic Acids Res. 44, W160-W165 (2016).

105. Kim, D., Langmead, B. \& Salzberg, S. L. HISAT: a fast spliced aligner with low memory requirements. Nat. Methods 12, 357-360 (2015).

106. Liao, Y., Smyth, G. K. \& Shi, W. The Subread aligner: fast, accurate and scalable read mapping by seed-and-vote. Nucleic Acids Res. 41, e108 (2013).

107. Love, M. I., Huber, W. \& Anders, S. Moderated estimation of fold change and dispersion for RNA-seq data with DESeq2. Genome Biol. 15, 550 (2014).

\section{Acknowledgements}

We thank the Developmental Studies Hybridoma Bank (DSHB) and Cintia Hongay for antibodies, Jean-Yves Roignant, Matthias Soller and the Bloomington Drosophila Stock Center (BDSC) for fly stocks, Stefan Ameres for the S2-S line and advice on cell mutagenesis. Sara Zaccara and Samie Jaffrey provided helpful advice on generation of miCLIP libraries and discussion about $\mathrm{m}^{6}$ A. A.C.-C. and S.O. were supported by grants from the Singapore Ministry of Education (MOE2013-T2-2-054 and MOE2017-T2-1089) and by Duke-NUS Medical School. S.O. was supported by a Khoo Postdoctoral Fellowship Award (Duke-NUS-KPFA/2017/0015). Work in the REK lab was supported by R01-GM132189. W.D. was generously supported by the Edward C. Taylor 3rd Year Graduate Fellowship in Chemistry. Work in the ECL lab was supported by NIH grants R01-GM083300 and R01-NS083833, and by the MSK Core Grant P30-CA008748.

\section{Author contributions}

L.K. generated all constructs and transgenic animals; constructed the miCLIP and RNAseq libraries; performed SUnSET, RNA degradation, and reporter assays, and all disc and CNS immunostainings. S.O. generated methods and software, conducted all the behavioral assays, and helped draft the manuscript. B.J. performed all the bioinformatic analyses. E.S.P. generated mettl3-KO cell lines and conducted co-IP and RIP tests. W.D. analyzed $\mathrm{m}^{6} \mathrm{~A}-\mathrm{YTH}$ factor interactions, performed proteomic analyses, and quantified modified nucleosides. A.C.-C., R.E.K., and E.C.L. supervised experiments in their respective laboratories, obtained funding, helped interpret analyses, and wrote the manuscript with input from all other authors. E.C.L. and A.C.-C. conceived the project.

\section{Competing interests}

The authors declare no competing interests.

\section{Additional information}

Supplementary information The online version contains supplementary material available at https://doi.org/10.1038/s41467-021-21537-1.

Correspondence and requests for materials should be addressed to E.C.L.

Peer review information Nature Communications thanks Alex Keene and the other anonymous reviewer(s) for their contribution to the peer review of this work. Peer reviewer reports are available.

Reprints and permission information is available at http://www.nature.com/reprints

Publisher's note Springer Nature remains neutral with regard to jurisdictional claims in published maps and institutional affiliations.

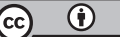

Open Access This article is licensed under a Creative Commons Attribution 4.0 International License, which permits use, sharing, adaptation, distribution and reproduction in any medium or format, as long as you give appropriate credit to the original author(s) and the source, provide a link to the Creative Commons license, and indicate if changes were made. The images or other third party material in this article are included in the article's Creative Commons license, unless indicated otherwise in a credit line to the material. If material is not included in the article's Creative Commons license and your intended use is not permitted by statutory regulation or exceeds the permitted use, you will need to obtain permission directly from the copyright holder. To view a copy of this license, visit http://creativecommons.org/ licenses/by/4.0/.

(c) The Author(s) 2021, corrected publication 2021 